\title{
Open charm and bottom tetraquarks in an extended relativized quark model
}

\author{
Qi-Fang Lü®, ${ }^{1,2,3, *}$ Dian-Yong Chen, ${ }^{4, \dagger}$ and Yu-Bing Dong ${ }^{5,6,7, 末}$ \\ ${ }^{1}$ Department of Physics, Hunan Normal University, Changsha 410081, China \\ ${ }^{2}$ Synergetic Innovation Center for Quantum Effects and Applications (SICQEA), Changsha 410081, China \\ ${ }^{3}$ Key Laboratory of Low-Dimensional Quantum Structures and Quantum Control of Ministry of Education, \\ Changsha 410081, China \\ ${ }^{4}$ School of Physics, Southeast University, Nanjing 210094, China \\ ${ }^{5}$ Institute of High Energy Physics, Chinese Academy of Sciences, Beijing 100049, China \\ ${ }^{6}$ Theoretical Physics Center for Science Facilities (TPCSF), CAS, Beijing 100049, China \\ ${ }^{7}$ School of Physical Sciences, University of Chinese Academy of Sciences, Beijing 101408, China
}

(Received 18 August 2020; accepted 23 September 2020; published 26 October 2020)

\begin{abstract}
In this work we systematically calculate the mass spectra of open charm and bottom tetraquarks $q q \bar{q} \bar{Q}$ within an extended relativized quark model. The four-body relativized Hamiltonians including the Coulomb potential, confining potential, spin-spin interactions, and relativistic corrections are solved by using the variational method. We find that the predicted masses of four $0^{+} u d \bar{s} \bar{c}$ states are 2765, 3065, 3152 , and $3396 \mathrm{MeV}$, which disfavor the assignment of the newly observed $X_{0}(2900)$ as a compact tetraquark. Moreover, the mass spectra of the open charm and bottom tetraquarks show quite similar patterns, which preserve the light flavor $\mathrm{SU}(3)$ symmetry and heavy quark symmetry well. In addition, our results suggest that the flavor-exotic states $n n \bar{s} \bar{c}, n n \bar{s} \bar{b}, s s \bar{n} \bar{c}$, and $s s \bar{n} \bar{b}$ and their antiparticles can be searched for in the heavy-light meson plus kaon final states by future experiments. More theoretical and experimental efforts are needed to investigate these singly heavy tetraquarks.
\end{abstract}

DOI: $10.1103 /$ PhysRevD.102.074021

\section{INTRODUCTION}

In the past years, many new hadronic states have been observed experimentally, and some of them cannot be simply assigned to the conventional mesons or baryons. This significant progress in experiments has triggered plenty of theoretical interest and made the study of these exotic states an intriguing topic in hadronic physics [1-14]. Among these states, the charged resonances $Z_{c(b)}[15-19]$, fully heavy tetraquark $\mathrm{X}(6900)$ [20], and pentaquarks $P_{c}$ $[21,22]$, are particularly interesting, since they cannot mix with traditional hadrons in the heavy quark sectors. Besides hidden charm and bottom states, the existence of flavorexotic states, where quarks and antiquarks cannot annihilate though strong and electromagnetic interactions, was also predicted. Therefore, searching for these flavor-exotic states has become increasingly important both theoretically and experimentally.

\footnotetext{
*1vqifang@hunnu.edu.cn †chendy@seu.edu.cn

†dongyb@ihep.ac.cn
}

Published by the American Physical Society under the terms of the Creative Commons Attribution 4.0 International license. Further distribution of this work must maintain attribution to the author(s) and the published article's title, journal citation, and DOI. Funded by SCOAP.
In 2016, the D0 Collaboration reported evidence of a narrow structure $X(5568)$, which is expected to be composed of four different flavors [23]. Unfortunately, its existence was not confirmed by the LHCb, CMS, CDF, and ATLAS collaborations [24-27], though the D0 Collaboration claimed that the $X(5568)$ was also found in different decay chains [28]. Before the observation of $X(5568)$, there existed a few studies on open charm and bottom tetraquark states, which mainly concentrated on the tetraquark interpretation of $D_{s 0}^{*}(2317)$ [29-35]. The evidence of $X(5568)$ immediately attracted great interest and many extensive theoretical investigations under various interpretations, such as a tetraquark [36-55], molecules [56-65], and kinematic effects [66-68]. Other related topics were also widely discussed [69-82], and the review on $X(5568)$ can be found in Ref. [4]. Basically, the experimental and theoretical efforts indicated that the $X(5568)$ should not be a genuine resonance. Although the searches of $X(5568)$ failed, the investigations of open charm and bottom tetraquark states have been revitalized.

Very recently, the $\mathrm{LHCb}$ Collaboration reported the observation of an exotic structure near $2.9 \mathrm{GeV}$ in the $D^{-} K^{+}$invariant mass spectrum via the $B^{+} \rightarrow D^{+} D^{-} K^{+}$ decay channel $[83,84]$. Then, this peak was modeled according to two resonances, $X_{0}(2900)$ and $X_{1}(2900)$. Their parameters are fitted to be 


$$
\begin{aligned}
m\left[X_{0}(2900)\right] & =2866.3 \pm 6.5 \pm 2.0 \mathrm{MeV}, \\
\Gamma\left[X_{0}(2900)\right] & =57.2 \pm 12.2 \pm 4.1 \mathrm{MeV}, \\
m\left[X_{1}(2900)\right] & =2904.1 \pm 4.8 \pm 1.3 \mathrm{MeV}, \\
\Gamma\left[X_{1}(2900)\right] & =110.3 \pm 10.7 \pm 4.3 \mathrm{MeV} .
\end{aligned}
$$

Given their $D^{-} K^{+}$decay mode, the quantum numbers of $X_{0}(2900)$ and $X_{1}(2900)$ should be $J^{P}=0^{+}$and $1^{-}$, respectively. Also, both of them have four different flavors, which indicates their exotic nature.

After the observation by the LHCb Collaboration, these two states near $2.9 \mathrm{GeV}$ were discussed within the simple quark model [85]. The $X_{0}(2900)$ was interpreted as an isosinglet compact tetraquark state, while the $X_{1}(2900)$ may be regarded as an artifact due to rescattering effects or a $J^{P}=2^{+} \bar{D}^{*} K^{*}$ molecule [85]. In fact, the $D^{*} \bar{K}^{*}$ molecules have been studied within a coupled channel unitary approach, and the predicted mass of the $J^{P}=0^{+}$state agrees well with the mass of the $X_{0}(2900)$ resonance [86]. Until now, no rigorous four-body calculation for these two states has been done, and all sorts of explanations are possible. Therefore, it is essential to investigate the possible compact tetraquark interpretations of $X_{0}(2900)$ and $X_{1}(2900)$ within realistic potentials.

In Refs. $[87,88]$ we extended the relativized quark model proposed by Godfrey and Isgur to investigate the doubly and fully heavy tetraquarks with the original model parameters. This extension allows us to describe the tetraquarks and conventional mesons in a uniform frame. Since the relativized potential can give a unified description of different flavor sectors and involve relativistic effects, it is believed to be more suitable to deal with the heavy-light and light-light quark interactions. In this work, we will systematically investigate the open charm and bottom tetraquarks $q q \bar{q} \bar{Q}$ in the extended relativized quark model and test the possible assignments of the newly observed resonances.

This paper is organized as follows. In Sec. II we briefly introduce the formalism of our extended relativized quark model for tetraquarks. Then, the mass spectra and discussions of our numerical results are presented in Sec. III. Finally, a short summary is given in the last section.

\section{EXTENDED RELATIVIZED QUARK MODEL}

To investigate the $S$-wave mass spectra of open charm and bottom tetraquarks $q_{1} q_{2} \bar{q}_{3} \bar{Q}_{4}$, the extended relativized quark model is employed [87]. This model is a natural generalization of the relativized quark model to deal with the tetraquark states. The relevant Hamiltonian with quark and gluon degrees of freedom for a $q_{1} q_{2} \bar{q}_{3} \bar{Q}_{4}$ state can be written as

$$
H=H_{0}+\sum_{i<j} V_{i j}^{\mathrm{oge}}+\sum_{i<j} V_{i j}^{\mathrm{conf}}
$$

where

$$
H_{0}=\sum_{i=1}^{4}\left(p_{i}^{2}+m_{i}^{2}\right)^{1 / 2}
$$

is the relativistic kinetic energy, $V_{i j}^{\text {oge }}$ is the one-gluonexchange potential together with the spin-spin interactions, and $V_{i j}^{\text {conf }}$ corresponds to the confinement potential. The useful formulas and parameters of these relativized potentials are presented in the Appendix, and the details can be found in Refs. [87,89].

The wave function for a $q_{1} q_{2} \bar{q}_{3} \bar{Q}_{4}$ state consists of four different parts: color, flavor, spin, and spatial wave functions. For the color part, there are two types of colorless states with certain permutation properties,

$$
\begin{aligned}
& |\overline{3} 3\rangle=\left|\left(q_{1} q_{2}\right)^{\overline{3}}\left(\bar{q}_{3} \bar{Q}_{4}\right)^{3}\right\rangle, \\
& |6 \overline{6}\rangle=\left|\left(q_{1} q_{2}\right)^{6}\left(\bar{q}_{3} \bar{Q}_{4}\right)^{\overline{6}}\right\rangle .
\end{aligned}
$$

Here, the $|\overline{3} 3\rangle$ and $|6 \overline{6}\rangle$ are antisymmetric and symmetric color wave functions under the exchange of $q_{1} q_{2}$ or $\bar{q}_{3} \bar{Q}_{4}$, respectively. For the flavor part, the combination $q_{1} q_{2}$ can be either symmetric or antisymmetric, while the $\bar{q}_{3}$ and $\bar{Q}_{4}$ are treated as different particles without symmetry constraints. To distinguish the up, down, and strange quarks clearly, we adopt the notation " $n$ " to stand for the up or down quark, and " $s$ " to represent the strange quark.

In the spin space, the six spin basis states can be expressed as

$$
\begin{aligned}
& \chi_{0}^{00}=\left|\left(q_{1} q_{2}\right)_{0}\left(\bar{q}_{3} \bar{Q}_{4}\right)_{0}\right\rangle_{0}, \\
& \chi_{0}^{11}=\left|\left(q_{1} q_{2}\right)_{1}\left(\bar{q}_{3} \bar{Q}_{4}\right)_{1}\right\rangle_{0}, \\
& \chi_{1}^{01}=\left|\left(q_{1} q_{2}\right)_{0}\left(\bar{q}_{3} \bar{Q}_{4}\right)_{1}\right\rangle_{1}, \\
& \chi_{1}^{10}=\left|\left(q_{1} q_{2}\right)_{1}\left(\bar{q}_{3} \bar{Q}_{4}\right)_{0}\right\rangle_{1}, \\
& \chi_{1}^{11}=\left|\left(q_{1} q_{2}\right)_{1}\left(\bar{q}_{3} \bar{Q}_{4}\right)_{1}\right\rangle_{1}, \\
& \chi_{2}^{11}=\left|\left(q_{1} q_{2}\right)_{1}\left(\bar{q}_{3} \bar{Q}_{4}\right)_{1}\right\rangle_{2},
\end{aligned}
$$

where $\left(q_{1} q_{2}\right)_{0}$ and $\left(\bar{q}_{3} \bar{Q}_{4}\right)_{0}$ are antisymmetric for the two fermions under permutations, while the $\left(q_{1} q_{2}\right)_{1}$ and $\left(\bar{q}_{3} \bar{Q}_{4}\right)_{1}$ are symmetric ones. The relevant matrix elements of the color and spin parts for various types of tetraquark states are identical [87].

In the spatial space, the Jacobi coordinates are shown in Fig. 1. For a $q_{1} q_{2} \bar{q}_{3} \bar{Q}_{4}$ state, one can define

$$
\begin{aligned}
& \boldsymbol{r}_{12}=\boldsymbol{r}_{1}-\boldsymbol{r}_{2}, \\
& \boldsymbol{r}_{34}=\boldsymbol{r}_{3}-\boldsymbol{r}_{4},
\end{aligned}
$$




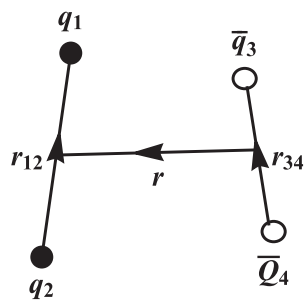

(a)

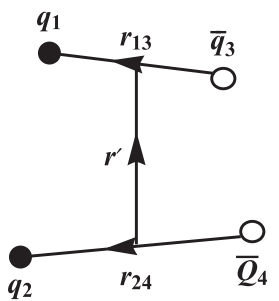

(b)

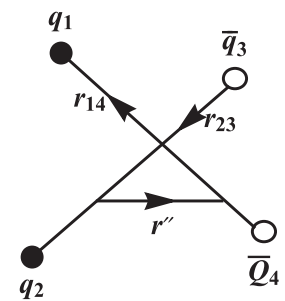

(c)

FIG. 1. The $q_{1} q_{2} \bar{q}_{3} \bar{Q}_{4}$ tetraquark state in Jacobi coordinates. (a) combination $\left(q_{1} q_{2}\right)\left(\bar{q}_{3} \bar{Q}_{4}\right)$ with relative coordinates $\boldsymbol{r}_{12}$, $\boldsymbol{r}_{34}$, and $\boldsymbol{r}$, (b) combination $\left(q_{1} \bar{q}_{3}\right)\left(q_{2} \bar{Q}_{4}\right)$ with relative coordinates $\boldsymbol{r}_{13}, \boldsymbol{r}_{24}$, and $\boldsymbol{r}^{\prime}$, (c) combination $\left(q_{1} \bar{Q}_{4}\right)\left(q_{2} \bar{q}_{3}\right)$ with relative coordinates $\boldsymbol{r}_{14}, \boldsymbol{r}_{23}$, and $\boldsymbol{r}^{\prime \prime}$.

$$
\boldsymbol{r}=\frac{m_{1} \boldsymbol{r}_{1}+m_{2} \boldsymbol{r}_{2}}{m_{1}+m_{2}}-\frac{m_{3} \boldsymbol{r}_{3}+m_{4} \boldsymbol{r}_{4}}{m_{3}+m_{4}},
$$

and

$$
\boldsymbol{R}=\frac{m_{1} \boldsymbol{r}_{1}+m_{2} \boldsymbol{r}_{2}+m_{3} \boldsymbol{r}_{3}+m_{4} \boldsymbol{r}_{4}}{m_{1}+m_{2}+m_{3}+m_{4}} .
$$

Then, any other relative coordinates of this system can be expressed in terms of $\boldsymbol{r}_{12}, \boldsymbol{r}_{34}$, and $\boldsymbol{r}$ [87]. A set of Gaussian functions is adopted to approach the $S$-wave realistic spatial wave function [90]

$\Psi\left(\boldsymbol{r}_{12}, \boldsymbol{r}_{34}, \boldsymbol{r}\right)=\sum_{n_{12}, n_{34}, n} C_{n_{12} n_{34} n} \psi_{n_{12}}\left(\boldsymbol{r}_{12}\right) \psi_{n_{34}}\left(\boldsymbol{r}_{34}\right) \psi_{n}(\boldsymbol{r})$,

where $C_{n_{12} n_{34} n}$ are the expansion coefficients. The $\psi_{n_{12}}\left(\boldsymbol{r}_{12}\right) \psi_{n_{34}}\left(\boldsymbol{r}_{34}\right) \psi_{n}(\boldsymbol{r})$ is the position representation of the basis $\left|n_{12} n_{34} n\right\rangle$, where

$$
\psi_{n}(\boldsymbol{r})=\frac{2^{7 / 4} \nu_{n}^{3 / 4}}{\pi^{1 / 4}} e^{-\nu_{n} r^{2}} Y_{00}(\hat{\boldsymbol{r}})=\left(\frac{2 \nu_{n}}{\pi}\right)^{3 / 4} e^{-\nu_{n} r^{2}},
$$

$$
\nu_{n}=\frac{1}{r_{1}^{2} a^{2(n-1)}}, \quad\left(n=1-N_{\max }\right)
$$

The final results are independent with geometric Gaussian size parameters $r_{1}, a$, and $N_{\max }$ when adequate bases are chosen [90]. The $\psi_{n_{12}}\left(\boldsymbol{r}_{12}\right)$ and $\psi_{n_{34}}\left(\boldsymbol{r}_{34}\right)$ can be expressed in a similar way, and the momentum representation of the basis $\left|n_{12} n_{34} n\right\rangle$ can be obtained via Fourier transformation. The numerical error of our approach has been analyzed in Ref. [87], which is sufficient for quark model predictions.

According to the Pauli exclusion principle, the wave function of a tetraquark should be antisymmetric for the identical quarks and antiquarks. All possible configurations for the $q_{1} q_{2} \bar{q}_{3} \bar{Q}_{4}$ systems are listed in Table I. The subscripts and superscripts are the spin and color representations, respectively. The brackets [] and braces \{\} stand for the antisymmetric and symmetric flavor wave functions, respectively. The parentheses () are adopted for the subsystems without permutation symmetries.

With the total wave functions, all of the matrix elements

\begin{tabular}{|c|c|c|c|c|}
\hline System & $I\left(J^{P}\right)$ & & Configuration & \\
\hline \multirow[t]{3}{*}[nn]{$(\bar{n} \bar{c})$} & $\frac{1}{2}\left(0^{+}\right)$ & $\left|[n n]_{0}^{\overline{3}}(\bar{n} \bar{c})_{0}^{3}\right\rangle_{0}$ & $\left|[n n]_{1}^{6}(\bar{n} \bar{c})_{1}^{\overline{6}}\right\rangle_{0}$ & \\
\hline & $\frac{1}{2}\left(1^{+}\right)$ & $\left|[n n]_{0}^{\overline{3}}(\bar{n} \bar{c})_{\underline{1}}^{3}\right\rangle_{1}$ & $\left|[n n]_{1}^{6}(\bar{n} \bar{c})_{0}^{\overline{6}}\right\rangle_{1}$ & $\left|[n n]_{1}^{6}(\bar{n} \bar{c})_{1}^{\overline{6}}\right\rangle_{1}$ \\
\hline & $\frac{1}{2}\left(2^{+}\right)$ & $\left|[n n]_{1}^{6}(\bar{n} \bar{c})_{1}^{\overline{6}}\right\rangle_{2}$ & $\cdots$ & \\
\hline \multirow{3}{*}{$n n$}$(\bar{n} \bar{c})$ & $\frac{1}{2} / \frac{3}{2}\left(0^{+}\right)$ & $\left|\{n n\}_{1}^{\overline{3}}(\bar{n} \bar{c})_{1}^{3}\right\rangle_{0}$ & $\left|\{n n\}_{0}^{6}(\bar{n} \bar{c})_{0}^{\bar{\sigma}}\right\rangle_{0}$ & \\
\hline & $\frac{1}{2} / \frac{3}{2}\left(1^{+}\right)$ & $\left|\{n n\}_{1}^{\frac{1}{3}}(\bar{n} \bar{c})_{0}^{3}\right\rangle_{1}$ & $\left|\{n n\}_{1}^{3}(\bar{n} \bar{c})_{1}^{3}\right\rangle_{1}$ & $\left|\{n n\}_{0}^{6}(\bar{n} \bar{c})_{1}^{\overline{6}}\right\rangle_{1}$ \\
\hline & $\frac{1}{2} / \frac{3}{2}\left(2^{+}\right)$ & $\left|\{n n\}_{1}^{\overline{3}}(\bar{n} \bar{c})_{1}^{3}\right\rangle_{2}$ & $\cdots$ & $\cdots$ \\
\hline \multirow[t]{3}{*}[nn]{$(\bar{n} \bar{b})$} & $\frac{1}{2}\left(0^{+}\right)$ & $\left|[n n]_{0}^{3}(\bar{n} \bar{b})_{0}^{3}\right\rangle_{0}$ & $\left|[n n]_{1}^{6}(\bar{n} \bar{b})_{1}^{\overline{6}}\right\rangle_{0}$ & $\cdots$ \\
\hline & $\frac{1}{2}\left(1^{+}\right)$ & $\left|[n n]_{0}^{\overline{3}}(\bar{n} \bar{b})_{1}^{3}\right\rangle_{1}$ & $\left|[n n]_{1}^{6}(\bar{n} \bar{b})_{0}^{\overline{6}}\right\rangle_{1}$ & $\left|[n n]_{1}^{6}(\bar{n} \bar{b})_{1}^{\overline{6}}\right\rangle_{1}$ \\
\hline & $\frac{1}{2}\left(2^{+}\right)$ & $\left|[n n]_{1}^{6}(\bar{n} \bar{b})_{1}^{\overline{6}}\right\rangle_{2}$ & $\cdots$ & $\ldots$ \\
\hline \multirow[t]{3}{*}{$n n$}$(\bar{n} \bar{b})$ & $\frac{1}{2} / \frac{3}{2}\left(0^{+}\right)$ & $\left|\{n n\}_{1}^{3}(\bar{n} \bar{b})_{1}^{3}\right\rangle_{0}$ & $\left|\{n n\}_{0}^{6}(\bar{n} \bar{b})_{0}^{\overline{6}}\right\rangle_{0}$ & $\ldots$ \\
\hline & $\frac{1}{2} / \frac{3}{2}\left(1^{+}\right)$ & $\left|\{n n\}_{1}^{\overline{3}}(\bar{n} \bar{b})_{0}^{3}\right\rangle_{1}$ & $\left|\{n n\}_{1}^{\overline{3}}(\bar{n} \bar{b})_{1}^{3}\right\rangle_{1}$ & $\left|\{n n\}_{0}^{6}(\bar{n} \bar{b})_{1}^{\overline{6}}\right\rangle_{1}$ \\
\hline & $\frac{1}{2} / \frac{3}{2}\left(2^{+}\right)$ & $\left|\{n n\}_{1}^{3}(\bar{n} \bar{b})_{1}^{3}\right\rangle_{2}$ & & \\
\hline
\end{tabular}
involved in the Hamiltonian can be worked out straightforwardly. The masses without a mixing mechanism

TABLE I. All possible configurations for the $q_{1} q_{2} \bar{q}_{3} \bar{Q}_{4}$ systems. 
TABLE I. (Continued)

\begin{tabular}{|c|c|c|c|c|}
\hline System & $I\left(J^{P}\right)$ & & Configuration & \\
\hline$[n n](\bar{s} \bar{c})$ & $\begin{array}{l}0\left(0^{+}\right) \\
0\left(1^{+}\right) \\
0\left(2^{+}\right)\end{array}$ & $\begin{array}{l}\left|[n n]_{0}^{\overline{3}}(\bar{s} \bar{c})_{0}^{3}\right\rangle_{0} \\
\mid[n n]_{0}^{\overline{3}}\left(\left.\bar{s} \bar{c}\right|_{1} ^{3}\right\rangle_{1} \\
\left|[n n]_{1}^{6}(\bar{s} \bar{c})_{1}^{6}\right\rangle_{2}\end{array}$ & $\begin{array}{l}\left|[n n]_{1}^{6}(\bar{s} \bar{c})_{1}^{\overline{6}}\right\rangle_{0} \\
\left|[n n]_{1}^{6}(\bar{s} \bar{c})_{0}^{6}\right\rangle_{1}\end{array}$ & $\left|[n n]_{1}^{6}(\bar{s} \bar{c})_{1}^{\overline{6}}\right\rangle_{1}$ \\
\hline$\{n n\}(\bar{s} \bar{c})$ & $\begin{array}{l}1\left(0^{+}\right) \\
1\left(1^{+}\right) \\
1\left(2^{+}\right)\end{array}$ & $\begin{array}{l}\left|\{n n\}_{1}^{\overline{3}}(\bar{s} \bar{c})_{1}^{3}\right\rangle_{0} \\
\left|\{n n\}_{1}^{3}(\bar{s} \bar{c})_{0}^{3}\right\rangle_{1} \\
\left|\{n n\}_{1}^{3}(\bar{s} \bar{c})_{1}^{3}\right\rangle_{2}\end{array}$ & $\begin{array}{l}\left|\{n n\}_{0}^{6}(\bar{s} \bar{c})_{0}^{\overline{6}}\right\rangle_{0} \\
\left|\{n n\}_{1}^{\overline{3}}(\bar{s} \bar{c})_{1}^{3}\right\rangle_{1}\end{array}$ & $\left|\{n n\}_{0}^{6}(\bar{s} \bar{c})_{1}^{\overline{6}}\right\rangle_{1}$ \\
\hline$[n n](\bar{s} \bar{b})$ & $\begin{array}{l}0\left(0^{+}\right) \\
0\left(1^{+}\right) \\
0\left(2^{+}\right)\end{array}$ & $\begin{array}{l}\left|[n n]_{0}^{\overline{3}}(\bar{s} \bar{b})_{0}^{3}\right\rangle_{0} \\
\left|[n n]_{0}^{\overline{3}}(\bar{s} \bar{b})_{1}^{3}\right\rangle_{1} \\
\left|[n n]_{1}^{6}(\bar{s} \bar{b})_{1}^{6}\right\rangle_{2}\end{array}$ & $\begin{array}{c}\left|[n n]_{1}^{6}(\bar{s} \bar{b})_{1}^{\overline{6}}\right\rangle_{0} \\
\left|[n n]_{1}^{6}(\bar{s} \bar{b})_{0}^{6}\right\rangle_{1} \\
\cdots\end{array}$ & $\begin{array}{c}\left|[n n]_{1}^{6}(\bar{s} \bar{b})_{1}^{\overline{6}}\right\rangle_{1} \\
\ldots\end{array}$ \\
\hline$\{n n\}(\bar{s} \bar{b})$ & $\begin{array}{l}1\left(0^{+}\right) \\
1\left(1^{+}\right) \\
1\left(2^{+}\right)\end{array}$ & $\begin{array}{l}\left|\{n n\}_{1}^{3}(\bar{s} \bar{b})_{1}^{3}\right\rangle_{0} \\
\left|\{n n\}_{1}^{3}(\bar{s} \bar{b})_{0}^{3}\right\rangle_{1} \\
\left|\{n n\}_{1}^{3}(\bar{s} \bar{b})_{1}^{3}\right\rangle_{2}\end{array}$ & $\begin{array}{l}\left|\{n n\}_{0}^{6}(\bar{s} \bar{b})_{0}^{\overline{6}}\right\rangle_{0} \\
\left|\{n n\}_{1}^{3}(\bar{s} \bar{b})_{1}^{3}\right\rangle_{1}\end{array}$ & $\left|\{n n\}_{0}^{6}(\bar{s} \bar{b})_{1}^{\overline{6}}\right\rangle_{1}$ \\
\hline$[n s](\bar{n} \bar{c})$ & $\begin{array}{l}0 / 1\left(0^{+}\right) \\
0 / 1\left(1^{+}\right) \\
0 / 1\left(2^{+}\right)\end{array}$ & $\begin{array}{l}\left|[n s]_{0}^{\overline{3}}(\bar{n} \bar{c})_{0}^{3}\right\rangle_{0} \\
\left|[n s]_{0}^{\overline{3}}(\bar{n} \bar{c})_{1}^{3}\right\rangle_{1} \\
\left|[n s]_{1}^{6}(\bar{n} \bar{c})_{1}^{6}\right\rangle_{2}\end{array}$ & $\begin{array}{l}\left|[n s]_{1}^{6}(\bar{n} \bar{c})_{1}^{\overline{6}}\right\rangle_{0} \\
\left|[n s]_{1}^{6}(\bar{n} \bar{c})_{0}^{\overline{6}}\right\rangle_{1}\end{array}$ & $\left|[n s]_{1}^{6}(\bar{n} \bar{c})_{1}^{\overline{6}}\right\rangle_{1}$ \\
\hline$\{n s\}(\bar{n} \bar{c})$ & $\begin{array}{l}0 / 1\left(0^{+}\right) \\
0 / 1\left(1^{+}\right) \\
0 / 1\left(2^{+}\right)\end{array}$ & $\begin{array}{l}\left|\{n s\}_{1}^{\overline{3}}(\bar{n} \bar{c})_{1}^{3}\right\rangle_{0} \\
\left|\{n s\}_{1}^{3}(\bar{n} \bar{c})_{0}^{3}\right\rangle_{1} \\
\left|\{n s\}_{1}^{3}(\bar{n} \bar{c})_{1}^{3}\right\rangle_{2}\end{array}$ & $\begin{array}{l}\left|\{n s\}_{0}^{6}(\bar{n} \bar{c})_{0}^{\overline{6}}\right\rangle_{0} \\
\left|\{n s\}_{1}^{\overline{3}}(\bar{n} \bar{c})_{1}^{3}\right\rangle_{1}\end{array}$ & $\left|\{n s\}_{0}^{6}(\bar{n} \bar{c})_{1}^{\overline{6}}\right\rangle_{1}$ \\
\hline$[n s](\bar{n} \bar{b})$ & $\begin{array}{l}0 / 1\left(0^{+}\right) \\
0 / 1\left(1^{+}\right) \\
0 / 1\left(2^{+}\right)\end{array}$ & $\begin{array}{l}\left|[n s]_{0}^{\overline{3}}(\bar{n} \bar{b})_{0}^{3}\right\rangle_{0} \\
\left|[n s]_{0}^{\overline{3}}(\bar{n} \bar{b})_{1}^{3}\right\rangle_{1} \\
\left|[n s]_{1}^{6}(\bar{n} \bar{b})_{1}^{6}\right\rangle_{2}\end{array}$ & $\begin{array}{c}\left|[n s]_{1}^{6}(\bar{n} \bar{b})_{1}^{\overline{6}}\right\rangle_{0} \\
\left|[n s]_{1}^{6}(\bar{n} \bar{b})_{0}^{6}\right\rangle_{1} \\
\ldots\end{array}$ & $\begin{array}{c}\ldots \\
\left|[n s]_{1}^{6}(\bar{n} \bar{b})_{1}^{\overline{6}}\right\rangle_{1} \\
\cdots\end{array}$ \\
\hline$\{n s\}(\bar{n} \bar{b})$ & $\begin{array}{l}0 / 1\left(0^{+}\right) \\
0 / 1\left(1^{+}\right) \\
0 / 1\left(2^{+}\right)\end{array}$ & $\begin{array}{l}\left|\{n s\}_{1}^{3}(\bar{n} \bar{b})_{1}^{3}\right\rangle_{0} \\
\left|\{n s\}_{1}^{3}(\bar{n} \bar{b})_{0}^{3}\right\rangle_{1} \\
\left|\{n s\}_{1}^{\overline{3}}(\bar{n} \bar{b})_{1}^{3}\right\rangle_{2}\end{array}$ & $\begin{array}{l}\left|\{n s\}_{0}^{6}(\bar{n} \bar{b})_{0}^{\overline{6}}\right\rangle_{0} \\
\left|\{n s\}_{1}^{\overline{3}}(\bar{n} \bar{b})_{1}^{3}\right\rangle_{1}\end{array}$ & $\left|\{n s\}_{0}^{6}(\bar{n} \bar{b})_{1}^{\overline{6}}\right\rangle_{1}$ \\
\hline$[n s](\bar{s} \bar{c})$ & $\begin{array}{l}\frac{1}{2}\left(0^{+}\right) \\
\frac{1}{2}\left(1^{+}\right) \\
\frac{1}{2}\left(2^{+}\right)\end{array}$ & $\begin{array}{l}\left|[n s]_{0}^{\overline{3}}(\bar{s} \bar{c})_{0}^{3}\right\rangle_{0} \\
\left|[n s]_{0}^{\overline{3}}(\bar{s} \bar{c})_{1}^{3}\right\rangle_{1} \\
\left|[n s]_{1}^{6}(\bar{s} \bar{c})_{1}^{6}\right\rangle_{2}\end{array}$ & $\begin{array}{l}\left|[n s]_{1}^{6}(\bar{s} \bar{c})_{1}^{\overline{6}}\right\rangle_{0} \\
\left|[n s]_{1}^{6}(\bar{s} \bar{c})_{0}^{\overline{6}}\right\rangle_{1}\end{array}$ & $\left|[n s]_{1}^{6}(\bar{s} \bar{c})_{1}^{\overline{6}}\right\rangle_{1}$ \\
\hline$\{n s\}(\bar{s} \bar{c})$ & $\begin{array}{l}\frac{1}{2}\left(0^{+}\right) \\
\frac{1}{2}\left(1^{+}\right) \\
\frac{1}{2}\left(2^{+}\right)\end{array}$ & $\begin{array}{l}\left|\{n s\}_{1}^{3}(\bar{s} \bar{c})_{1}^{3}\right\rangle_{0} \\
\left|\{n s\}_{1}^{3}(\bar{s} \bar{c})_{0}^{3}\right\rangle_{1} \\
\left|\{n s\}_{1}^{3}(\bar{s} \bar{c})_{1}^{3}\right\rangle_{2}\end{array}$ & $\begin{array}{c}\left|\{n s\}_{0}^{6}(\bar{s} \bar{c})_{0}^{6}\right\rangle_{0} \\
\left|\{n s\}_{1}^{3}(\bar{s} \bar{c})_{1}^{3}\right\rangle_{1} \\
\ldots\end{array}$ & $\begin{array}{c}\left|\{n s\}_{0}^{6}(\bar{s} \bar{c})_{1}^{\overline{6}}\right\rangle_{1} \\
\ldots\end{array}$ \\
\hline$[n s](\bar{s} \bar{b})$ & $\begin{array}{l}\frac{1}{2}\left(0^{+}\right) \\
\frac{1}{2}\left(1^{+}\right) \\
\frac{1}{2}\left(2^{+}\right)\end{array}$ & $\begin{array}{l}\left|[n s]_{0}^{\overline{3}}(\bar{s} \bar{b})_{0}^{3}\right\rangle_{0} \\
\left|[n s]_{0}^{\overline{3}}(\bar{s} \bar{b}]_{1}^{3}\right\rangle_{1} \\
\left|[n s]_{1}^{6}(\bar{s} \bar{b})_{1}^{6}\right\rangle_{2}\end{array}$ & $\begin{array}{c}\left|[n s]_{1}^{6}(\bar{s} \bar{b})_{1}^{\overline{6}}\right\rangle_{0} \\
\left|[n s]_{1}^{6}(\bar{s} \bar{b})_{0}^{6}\right\rangle_{1} \\
\ldots\end{array}$ & $\begin{array}{c}\ldots \\
\left|[n s]_{1}^{6}(\bar{s} \bar{b})_{1}^{\overline{6}}\right\rangle_{1} \\
\cdots\end{array}$ \\
\hline$\{n s\}(\bar{s} \bar{b})$ & $\begin{array}{l}\frac{1}{2}\left(0^{+}\right) \\
\frac{1}{2}\left(1^{+}\right) \\
\frac{1}{2}\left(2^{+}\right)\end{array}$ & $\begin{array}{l}\left|\{n s\}_{1}^{3}(\bar{s} \bar{b})_{1}^{3}\right\rangle_{0} \\
\left|\{n s\}_{1}^{3}(\bar{s} \bar{b})_{0}^{3}\right\rangle_{1} \\
\left|\{n s\}_{1}^{3}(\bar{s} \bar{b})_{1}^{3}\right\rangle_{2}\end{array}$ & $\begin{array}{l}\left|\{n s\}_{0}^{6}(\bar{s} \bar{b})_{0}^{\overline{6}}\right\rangle_{0} \\
\left|\{n s\}_{1}^{3}(\bar{s} \bar{b})_{1}^{3}\right\rangle_{1}\end{array}$ & $\left|\{n s\}_{0}^{6}(\bar{s} \bar{b})_{1}^{\overline{6}}\right\rangle_{1}$ \\
\hline$\{s s\}(\bar{n} \bar{c})$ & $\begin{array}{l}\frac{1}{2}\left(0^{+}\right) \\
\frac{1}{2}\left(1^{+}\right) \\
\frac{1}{2}\left(2^{+}\right)\end{array}$ & $\begin{array}{l}\left|\{s s\}_{1}^{\overline{3}}(\bar{n} \bar{c})_{1}^{3}\right\rangle_{0} \\
\left|\{s s\}_{1}^{\overline{3}}(\bar{n} \bar{c})_{0}^{3}\right\rangle_{1} \\
\left|\{s s\}_{1}^{\overline{3}}(\bar{n} \bar{c})_{1}^{3}\right\rangle_{2}\end{array}$ & $\begin{array}{l}\left|\{s s\}_{0}^{6}(\bar{n} \bar{c})_{0}^{\overline{6}}\right\rangle_{0} \\
\left|\{s s\}_{1}^{\overline{3}}(\bar{n} \bar{c})_{1}^{3}\right\rangle_{1}\end{array}$ & $\left|\{s s\}_{0}^{6}(\bar{n} \bar{c})_{1}^{\overline{6}}\right\rangle_{1}$ \\
\hline$\{s s\}(\bar{n} \bar{b})$ & $\begin{array}{l}\frac{1}{2}\left(0^{+}\right) \\
\frac{1}{2}\left(1^{+}\right) \\
\frac{1}{2}\left(2^{+}\right)\end{array}$ & $\begin{array}{l}\left|\{s s\}_{1}^{\overline{3}}(\bar{n} \bar{b})_{1}^{3}\right\rangle_{0} \\
\left|\{s s\}_{1}^{3}(\bar{n} \bar{b})_{0}^{3}\right\rangle_{1} \\
\left|\{s s\}_{1}^{\overline{3}}(\bar{n} \bar{b})_{1}^{3}\right\rangle_{2}\end{array}$ & $\begin{array}{l}\left|\{s s\}_{0}^{6}(\bar{n} \bar{b})_{0}^{\overline{6}}\right\rangle_{0} \\
\left|\{s s\}_{1}^{\overline{3}}(\bar{n} \bar{b})_{1}^{3}\right\rangle_{1}\end{array}$ & $\begin{array}{c}\left|\{s s\}_{0}^{6}(\bar{n} \bar{b})_{1}^{\overline{6}}\right\rangle_{1} \\
\cdots\end{array}$ \\
\hline$\{s s\}(\bar{s} \bar{c})$ & $\begin{array}{l}0\left(0^{+}\right) \\
0\left(1^{+}\right) \\
0\left(2^{+}\right)\end{array}$ & $\begin{array}{l}\left|\{s s\}_{1}^{\overline{3}}(\bar{s} \bar{c})_{1}^{3}\right\rangle_{0} \\
\left|\{s s\}_{1}^{3}(\bar{s} \bar{c})_{0}^{3}\right\rangle_{1} \\
\left|\{s s\}_{1}^{\overline{3}}(\bar{s} \bar{c})_{1}^{3}\right\rangle_{2}\end{array}$ & $\begin{array}{l}\left|\{s s\}_{0}^{6}(\bar{s} \bar{c})_{0}^{\overline{6}}\right\rangle_{0} \\
\left|\{s s\}_{1}^{\overline{3}}(\bar{s} \bar{c})_{1}^{3}\right\rangle_{1}\end{array}$ & $\begin{array}{c}\ldots \\
\left|\{s s\}_{0}^{6}(\bar{s} \bar{c})_{1}^{\overline{6}}\right\rangle_{1} \\
\ldots\end{array}$ \\
\hline
\end{tabular}


TABLE I. (Continued)

\begin{tabular}{llllc}
\hline \hline System & $I\left(J^{P}\right)$ & & Configuration & \\
\hline$\{s s\}(\bar{s} \bar{b})$ & $0\left(0^{+}\right)$ & $\left|\{s s\}_{1}^{\overline{3}}(\bar{s} \bar{b}){ }_{1}^{3}\right\rangle_{0}$ & $\left|\{s s\}_{0}^{6}(\bar{s} \bar{b})_{0}^{6}\right\rangle_{0}$ & $\cdots$ \\
& $0\left(1^{+}\right)$ & $\left|\{s s\}_{1}^{3}(\bar{s} \bar{b})_{0}^{3}\right\rangle_{1}$ & $\left|\{s s\}_{1}^{3}(\bar{s} \bar{b})_{1}^{3}\right\rangle_{1}$ & $\left|\{s s\}_{0}^{6}(\bar{s} \bar{b})_{1}^{\overline{6}}\right\rangle_{1}$ \\
& $0\left(2^{+}\right)$ & $\left|\{s s\}_{1}^{\overline{3}}(\bar{s} \bar{b})_{1}^{3}\right\rangle_{2}$ & $\cdots$ & $\cdots$ \\
\hline \hline
\end{tabular}

can be obtained by solving the generalized eigenvalue problem

$$
\sum_{j=1}^{N_{\max }^{3}}\left(H_{i j}-E N_{i j}\right) C_{j}=0, \quad\left(i=1-N_{\max }^{3}\right),
$$

where the $H_{i j}$ are the matrix elements of the Hamiltonian, $N_{i j}$ are the overlap matrix elements of the Gaussian bases due to their nonorthogonality, $E$ is the mass, and $C_{j}$ is the eigenvector corresponding to the expansion coefficients $C_{n_{12} n_{34} n}$ for the spatial wave function. For a given system, different configurations with the same $I\left(J^{P}\right)$ should mix with each other. The final mass spectra and wave functions are obtained by diagonalizing the mass matrix of these configurations.

\section{RESULTS AND DISCUSSIONS}

In this work, we adopt $N_{\max }^{3}=10^{3}$ Gaussian bases to calculate the mass spectra of $S$-wave $q_{1} q_{2} \bar{q}_{3} \bar{Q}_{4}$ tetraquark states systematically. Under these large bases, our numerical results are stable enough for quark model estimations. According to the number of strange quarks, one can classify these open charm and bottom tetraquarks into four groups. We will examine the mass spectra of these systems successively.

The predicted masses for the nonstrange tetraquarks $n n \bar{n} \bar{c}$ and $n n \bar{n} \bar{b}$ are presented in Table II. For the $n n \bar{n} \bar{c}$ system, the masses lie in the range $2570-3327 \mathrm{MeV}$, while the masses of $n n \bar{n} \bar{b}$ states vary from 5977 to $6621 \mathrm{MeV}$. It can be noticed that these mass regions have significant

TABLE II. Predicted mass spectra for the $n n \bar{n} \bar{c}$ and $n n \bar{n} \bar{b}$ systems.

\begin{tabular}{|c|c|c|c|c|}
\hline$I\left(J^{P}\right)$ & Configuration & $\langle H\rangle(\mathrm{MeV})$ & Mass $(\mathrm{MeV})$ & Eigenvector \\
\hline$\overline{\frac{1}{2}\left(0^{+}\right)}$ & $\begin{array}{l}\left|[n n]_{0}^{\overline{3}}(\bar{n} \bar{c})_{0}^{3}\right\rangle_{0} \\
\left|[n n]_{1}^{6}(\bar{n} \bar{c})_{0}^{6}\right\rangle_{0}\end{array}$ & $\left(\begin{array}{rr}2868 & -242 \\
-242 & 2766\end{array}\right)$ & {$\left[\begin{array}{l}2570 \\
3064\end{array}\right]$} & {$\left[\begin{array}{c}(0.630,0.776) \\
(0.776,-0.630)\end{array}\right]$} \\
\hline$\frac{1}{2}\left(1^{+}\right)$ & $\begin{array}{l}\left|[n n]_{0}^{\overline{3}}(\bar{n} \bar{c})_{1}^{3}\right\rangle_{1} \\
\left|[n n]_{1}^{6}(\bar{n} \bar{c})_{0}^{\overline{6}}\right\rangle_{1} \\
\left|[n n]_{1}^{6}(\bar{n} \bar{c})_{1}^{\overline{6}}\right\rangle_{1}\end{array}$ & $\left(\begin{array}{ccc}2930 & -112 & 77 \\
-112 & 3129 & 87 \\
77 & 87 & 2952\end{array}\right)$ & {$\left[\begin{array}{l}2802 \\
3019 \\
3190\end{array}\right]$} & {$\left[\begin{array}{c}(0.703,0.397,-0.590) \\
(0.632,0.032,0.774) \\
(-0.326,0.917,0.229)\end{array}\right]$} \\
\hline$\frac{1}{2}\left(2^{+}\right)$ & $\left|[n n]_{1}^{6}(\bar{n} \bar{c})_{1}^{6}\right\rangle_{2}$ & 3240 & 3240 & 1 \\
\hline$\frac{1}{2} / \frac{3}{2}\left(0^{+}\right)$ & $\begin{array}{l}\left|\{n n\}_{1}^{\overline{3}}(\bar{n} \bar{c})_{1}^{3}\right\rangle_{0} \\
\left|\{n n\}_{0}^{6}(\bar{n} \bar{c})_{0}^{6}\right\rangle_{0}\end{array}$ & $\left(\begin{array}{cc}3050 & 193 \\
193 & 3192\end{array}\right)$ & {$\left[\begin{array}{l}2915 \\
3327\end{array}\right]$} & {$\left[\begin{array}{c}(-0.820,0.572) \\
(-0.572,-0.820)\end{array}\right]$} \\
\hline$\frac{1}{2} / \frac{3}{2}\left(1^{+}\right)$ & $\begin{array}{l}\left|\{n n\}_{1}^{\overline{3}}(\bar{n} \bar{c})_{0}^{3}\right\rangle_{1} \\
\left|\{n n\}_{1}^{\overline{3}}(\bar{n} \bar{c})_{1}^{3}\right\rangle_{1} \\
\left|\{n n\}_{0}^{6}(\bar{n} \bar{c})_{1}^{\overline{6}}\right\rangle_{1}\end{array}$ & $\left(\begin{array}{ccc}3100 & -38 & 108 \\
-38 & 3107 & 70 \\
108 & 70 & 3173\end{array}\right)$ & {$\left[\begin{array}{l}2980 \\
3140 \\
3260\end{array}\right]$} & {$\left[\begin{array}{c}(0.662,0.504,-0.555) \\
(0.560,-0.824,-0.081) \\
(0.498,0.257,0.828)\end{array}\right]$} \\
\hline$\frac{1}{2} / \frac{3}{2}\left(2^{+}\right)$ & $\left|\{n n\}_{1}^{\overline{3}}(\bar{n} \bar{c})_{1}^{3}\right\rangle_{2}$ & 3210 & 3210 & 1 \\
\hline$\frac{1}{2}\left(0^{+}\right)$ & $\begin{array}{l}\left|[n n]_{0}^{\overline{3}}(\bar{n} \bar{b})_{0}^{3}\right\rangle_{0} \\
\left|[n n]_{1}^{6}(\bar{n} \bar{b})_{1}^{\overline{6}}\right\rangle_{0}\end{array}$ & $\left(\begin{array}{rr}6206 & -201 \\
-201 & 6153\end{array}\right)$ & {$\left[\begin{array}{l}5977 \\
6382\end{array}\right]$} & {$\left[\begin{array}{c}(0.659,0.752) \\
(0.752,-0.659)\end{array}\right]$} \\
\hline$\frac{1}{2}\left(1^{+}\right)$ & $\begin{array}{l}\left|[n n]_{0}^{\overline{3}}(\bar{n} \bar{b})_{1}^{3}\right\rangle_{1} \\
\left|[n n]_{1}^{6}(\bar{n} \bar{b})_{0}^{\overline{6}}\right\rangle_{1} \\
\left|[n n]_{1}^{6}(\bar{n} \bar{b})_{1}^{\overline{6}}\right\rangle_{1}\end{array}$ & $\left(\begin{array}{ccc}6230 & -97 & -113 \\
-97 & 6448 & -136 \\
-113 & -136 & 6308\end{array}\right)$ & {$\left[\begin{array}{l}6080 \\
6373 \\
6534\end{array}\right]$} & {$\left[\begin{array}{c}(-0.701,-0.402,-0.589) \\
(-0.706,0.275,0.653) \\
(0.101,-0.874,0.476)\end{array}\right]$} \\
\hline$\frac{1}{2}\left(2^{+}\right)$ & $\left|[n n]_{1}^{6}(\bar{n} \bar{b})_{1}^{6}\right\rangle_{2}$ & 6552 & 6552 & 1 \\
\hline$\frac{1}{2} / \frac{3}{2}\left(0^{+}\right)$ & $\begin{array}{l}\left|\{n n\}_{1}^{\overline{3}}(\bar{n} \bar{b})_{1}^{3}\right\rangle_{0} \\
\left|\{n n\}_{0}^{6}(\bar{n} \bar{b})_{0}^{6}\right\rangle_{0}\end{array}$ & $\left(\begin{array}{cc}6366 & 167 \\
167 & 6512\end{array}\right)$ & {$\left[\begin{array}{l}6256 \\
6621\end{array}\right]$} & {$\left[\begin{array}{c}(-0.836,0.548) \\
(-0.548,-0.836)\end{array}\right]$} \\
\hline$\frac{1}{2} / \frac{3}{2}\left(1^{+}\right)$ & $\begin{array}{l}\left|\{n n\}_{1}^{\overline{3}}(\bar{n} \bar{b})_{0}^{3}\right\rangle_{1} \\
\left|\{n n\}_{1}^{\overline{3}}(\bar{n} \bar{b})_{1}^{3}\right\rangle_{1} \\
\left|\{n n\}_{0}^{6}(\bar{n} \bar{b})_{1}^{\overline{6}}\right\rangle_{1}\end{array}$ & $\left(\begin{array}{ccc}6437 & -51 & 92 \\
-51 & 6415 & 102 \\
92 & 102 & 6503\end{array}\right)$ & {$\left[\begin{array}{l}6286 \\
6478 \\
6591\end{array}\right]$} & {$\left[\begin{array}{c}(0.546,0.643,-0.536) \\
(0.745,-0.666,-0.039) \\
(0.382,0.378,0.843)\end{array}\right]$} \\
\hline$\frac{1}{2} / \frac{3}{2}\left(2^{+}\right)$ & $\left|\{n n\}_{1}^{3}(\bar{n} \bar{b})_{1}^{3}\right\rangle_{2}$ & 6503 & 6503 & 1 \\
\hline
\end{tabular}


TABLE III. Predicted mass spectra for the $n n \bar{s} \bar{c}, n n \bar{s} \bar{b}, n s \bar{n} \bar{c}$, and $n s \bar{n} \bar{b}$ systems.

\begin{tabular}{|c|c|c|c|c|}
\hline$I\left(J^{P}\right)$ & Configuration & $\langle H\rangle(\mathrm{MeV})$ & Mass $(\mathrm{MeV})$ & Eigenvector \\
\hline $0\left(0^{+}\right)$ & $\begin{array}{l}\left|[n n]_{0}^{\overline{3}}(\bar{s} \bar{c})_{0}^{3}\right\rangle_{0} \\
\left|[n n]_{1}^{6}(\bar{s} \bar{c})_{1}^{\overline{6}}\right\rangle_{0}\end{array}$ & $\left(\begin{array}{cc}2975 & 193 \\
193 & 2942\end{array}\right)$ & {$\left[\begin{array}{l}2765 \\
3152\end{array}\right]$} & {$\left[\begin{array}{c}(-0.677,0.736) \\
(0.736,0.677)\end{array}\right]$} \\
\hline $0\left(1^{+}\right)$ & $\begin{array}{l}\left|[n n]_{0}^{\overline{3}}(\bar{s} \bar{c})_{1}^{3}\right\rangle_{1} \\
\left|[n n]_{1}^{6}(\bar{s} \bar{c})_{0}^{\overline{6}}\right\rangle_{1} \\
\left|[n n]_{1}^{6}(\bar{s} \bar{c})_{1}^{\overline{6}}\right\rangle_{1}\end{array}$ & $\left(\begin{array}{ccc}3027 & 91 & 43 \\
91 & 3223 & -44 \\
43 & -44 & 3085\end{array}\right)$ & {$\left[\begin{array}{l}2964 \\
3108 \\
3263\end{array}\right]$} & {$\left[\begin{array}{c}(-0.827,0.364,0.428) \\
(0.455,-0.015,0.891) \\
(-0.330,-0.931,0.153)\end{array}\right]$} \\
\hline $0\left(2^{+}\right)$ & $\left|[n n]_{1}^{6}(\bar{s} \bar{c})_{1}^{\overline{6}}\right\rangle_{2}$ & 3316 & 3316 & 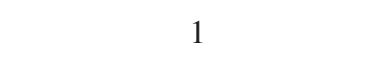 \\
\hline $1\left(0^{+}\right)$ & $\begin{array}{l}\left|\{n n\}_{1}^{\overline{3}}(\bar{s} \bar{c})_{1}^{3}\right\rangle_{0} \\
\left|\{n n\}_{0}^{6}(\bar{s} \bar{c})_{0}^{\overline{6}}\right\rangle_{0}\end{array}$ & $\left(\begin{array}{cc}3172 & -154 \\
-154 & 3289\end{array}\right)$ & {$\left[\begin{array}{l}3065 \\
3396\end{array}\right]$} & {$\left[\begin{array}{c}(-0.823,-0.568) \\
(0.568,-0.823)\end{array}\right]$} \\
\hline $1\left(1^{+}\right)$ & $\begin{array}{l}\left|\{n n\}_{1}^{\overline{3}}(\bar{s} \bar{c})_{0}^{3}\right\rangle_{1} \\
\left|\{n n\}_{1}^{3}(\bar{s} \bar{c})_{1}^{3}\right\rangle_{1} \\
\left|\{n n\}_{0}^{6}(\bar{s} \bar{c})_{1}^{6}\right\rangle_{1}\end{array}$ & $\left(\begin{array}{ccc}3211 & 23 & 87 \\
23 & 3218 & -40 \\
87 & -40 & 3275\end{array}\right)$ & {$\left[\begin{array}{l}3130 \\
3235 \\
3339\end{array}\right]$} & {$\left[\begin{array}{c}(-0.713,0.438,0.548) \\
(-0.457,-0.883,0.110) \\
(-0.532,0.171,-0.829)\end{array}\right]$} \\
\hline $1\left(2^{+}\right)$ & $\left|\{n n\}_{1}^{\overline{3}}(\bar{s} \bar{c})_{1}^{3}\right\rangle_{2}$ & 3302 & 3302 & 1 \\
\hline $0\left(0^{+}\right)$ & $\begin{array}{l}\left|[n n]_{0}^{\overline{3}}(\bar{s} \bar{b})_{0}^{3}\right\rangle_{0} \\
\left|[n n]_{1}^{6}(\bar{s} \bar{b})_{1}^{\overline{6}}\right\rangle_{0}\end{array}$ & $\left(\begin{array}{cc}6315 & 147 \\
147 & 6372\end{array}\right)$ & {$\left[\begin{array}{l}6194 \\
6493\end{array}\right]$} & {$\left[\begin{array}{c}(-0.771,0.636) \\
(-0.636,-0.771)\end{array}\right]$} \\
\hline $0\left(1^{+}\right)$ & $\begin{array}{l}\left|[n n]_{0}^{\overline{3}}(\bar{s} \bar{b})_{1}^{3}\right\rangle_{1} \\
\left|[n n]_{1}^{6}(\bar{s} \bar{b})_{0}^{\overline{6}}\right\rangle_{1} \\
\left|[n n]_{1}^{6}(\bar{s} \bar{b})_{1}^{\overline{6}}\right\rangle_{1}\end{array}$ & $\left(\begin{array}{ccc}6336 & -72 & -73 \\
-72 & 6580 & -84 \\
-73 & -84 & 6479\end{array}\right)$ & {$\left[\begin{array}{l}6272 \\
6492 \\
6630\end{array}\right]$} & {$\left[\begin{array}{c}(-0.848,-0.314,-0.427) \\
(-0.519,0.331,0.788) \\
(0.107,-0.890,0.443)\end{array}\right]$} \\
\hline $0\left(2^{+}\right)$ & $\left|[n n]_{1}^{6}(\bar{s} \bar{b})_{1}^{\overline{6}}\right\rangle_{2}$ & 6656 & 6656 & 1 \\
\hline $1\left(0^{+}\right)$ & $\begin{array}{l}\left|\{n n\}_{1}^{\overline{3}}(\bar{s} \bar{b})_{1}^{3}\right\rangle_{0} \\
\left|\{n n\}_{0}^{6}(\bar{s} \bar{b})_{0}^{\overline{6}}\right\rangle_{0}\end{array}$ & $\left(\begin{array}{cc}6499 & 122 \\
122 & 6645\end{array}\right)$ & {$\left[\begin{array}{l}6430 \\
6714\end{array}\right]$} & {$\left[\begin{array}{c}(-0.870,0.493) \\
(-0.493,-0.870)\end{array}\right]$} \\
\hline $1\left(1^{+}\right)$ & $\begin{array}{l}\left|\{n n\}_{1}^{\overline{3}}(\bar{s} \bar{b})_{0}^{3}\right\rangle_{1} \\
\left|\{n n\}_{1}^{\overline{3}}(\bar{s} \bar{b})_{1}^{3}\right\rangle_{1} \\
\left|\{n n\}_{0}^{6}(\bar{s} \bar{b})_{1}^{\overline{6}}\right\rangle_{1}\end{array}$ & $\left(\begin{array}{ccc}6549 & 35 & 68 \\
35 & 6535 & -66 \\
68 & -66 & 6639\end{array}\right)$ & {$\left[\begin{array}{l}6457 \\
6577 \\
6689\end{array}\right]$} & {$\left[\begin{array}{c}(0.592,-0.659,-0.463) \\
(-0.722,-0.689,0.059) \\
(0.358,-0.300,0.884)\end{array}\right]$} \\
\hline $1\left(2^{+}\right)$ & $\left|\{n n\}_{1}^{\overline{3}}(\bar{s} \bar{b})_{1}^{3}\right\rangle_{2}$ & 6602 & 6602 & 1 \\
\hline $0 / 1\left(0^{+}\right)$ & $\begin{array}{l}\left|[n s]_{0}^{\overline{3}}(\bar{n} \bar{c})_{0}^{3}\right\rangle_{0} \\
\left|[n s]_{1}^{6}(\bar{n} \bar{c})_{1}^{\overline{6}}\right\rangle_{0}\end{array}$ & $\left(\begin{array}{cc}3071 & -201 \\
-201 & 2960\end{array}\right)$ & {$\left[\begin{array}{l}2807 \\
3224\end{array}\right]$} & {$\left[\begin{array}{c}(0.606,0.795) \\
(0.795,-0.606)\end{array}\right]$} \\
\hline $0 / 1\left(1^{+}\right)$ & $\begin{array}{l}\left|[n s]_{0}^{\overline{3}}(\bar{n} \bar{c})_{1}^{3}\right\rangle_{1} \\
\left|[n s]_{1}^{6}(\bar{n} \bar{c})_{0}^{6}\right\rangle_{1} \\
\left|[n s]_{1}^{6}(\bar{n} \bar{c})_{1}^{\overline{6}}\right\rangle_{1}\end{array}$ & $\left(\begin{array}{ccc}3133 & 95 & 60 \\
95 & 3270 & -68 \\
60 & -68 & 3116\end{array}\right)$ & {$\left[\begin{array}{l}3013 \\
3181 \\
3325\end{array}\right]$} & {$\left[\begin{array}{c}(0.645,-0.409,-0.646) \\
(-0.656,0.138,-0.742) \\
(-0.392,-0.902,0.180)\end{array}\right]$} \\
\hline $0 / 1\left(2^{+}\right)$ & $\left|[n s]_{1}^{6}(\bar{n} \bar{c})_{1}^{\overline{6}}\right\rangle_{2}$ & 3364 & 3364 & 1 \\
\hline $0 / 1\left(0^{+}\right)$ & $\begin{array}{l}\left|\{n s\}_{1}^{\overline{3}}(\bar{n} \bar{c})_{1}^{3}\right\rangle_{0} \\
\left|\{n s\}_{0}^{6}(\bar{n} \bar{c})_{0}^{\overline{6}}\right\rangle_{0}\end{array}$ & $\left(\begin{array}{cc}3205 & -165 \\
-165 & 3317\end{array}\right)$ & {$\left[\begin{array}{l}3087 \\
3435\end{array}\right]$} & {$\left[\begin{array}{c}(-0.813,-0.583) \\
(0.583,-0.813)\end{array}\right]$} \\
\hline $0 / 1\left(1^{+}\right)$ & $\begin{array}{l}\left|\{n s\}_{1}^{\overline{3}}(\bar{n} \bar{c})_{0}^{3}\right\rangle_{1} \\
\left|\{n s\}_{1}^{\overline{3}}(\bar{n} \bar{c})_{1}^{3}\right\rangle_{1} \\
\left|\{n s\}_{0}^{6}(\bar{n} \bar{c})_{1}^{\overline{6}}\right\rangle_{1}\end{array}$ & $\left(\begin{array}{ccc}3236 & 30 & 93 \\
30 & 3253 & -56 \\
93 & -56 & 3297\end{array}\right)$ & {$\left[\begin{array}{l}3139 \\
3275 \\
3372\end{array}\right]$} & {$\left[\begin{array}{c}(0.685,-0.459,-0.566) \\
(-0.523,-0.851,0.057) \\
(0.507,-0.256,0.823)\end{array}\right]$} \\
\hline $0 / 1\left(2^{+}\right)$ & $\left|\{n s\}_{1}^{\overline{3}}(\bar{n} \bar{c})_{1}^{3}\right\rangle_{2}$ & 3339 & 3339 & 1 \\
\hline $0 / 1\left(0^{+}\right)$ & $\begin{array}{l}\left|[n s]_{0}^{\overline{3}}(\bar{n} \bar{b})_{0}^{3}\right\rangle_{0} \\
\left|[n s]_{1}^{6}(\bar{n} \bar{b})_{1}^{\overline{6}}\right\rangle_{0}\end{array}$ & $\left(\begin{array}{cc}6406 & 164 \\
164 & 6336\end{array}\right)$ & {$\left[\begin{array}{l}6203 \\
6538\end{array}\right]$} & {$\left[\begin{array}{c}(-0.629,0.778) \\
(0.778,0.629)\end{array}\right]$} \\
\hline $0 / 1\left(1^{+}\right)$ & $\begin{array}{l}\left|[n s]_{0}^{\frac{1}{3}}(\bar{n} \bar{b})_{1}^{3}\right\rangle_{1} \\
\left|[n s]_{1}^{6}(\bar{n} \bar{b})_{0}^{\overline{6}}\right\rangle_{1} \\
\left|[n s]_{1}^{6}(\bar{n} \bar{b})_{1}^{\overline{6}}\right\rangle_{1}\end{array}$ & $\left(\begin{array}{ccc}6430 & 81 & 91 \\
81 & 6581 & -110 \\
91 & -110 & 6462\end{array}\right)$ & {$\left[\begin{array}{l}6292 \\
6531 \\
6650\end{array}\right]$} & {$\left[\begin{array}{c}(-0.657,0.422,0.624) \\
(0.740,0.209,0.639) \\
(-0.139,-0.882,0.450)\end{array}\right]$} \\
\hline $0 / 1\left(2^{+}\right)$ & $\left|[n s]_{1}^{6}(\bar{n} \bar{b})_{1}^{\overline{6}}\right\rangle_{2}$ & 6668 & 6668 & 1 \\
\hline $0 / 1\left(0^{+}\right)$ & $\begin{array}{l}\left|\{n s\}_{1}^{\overline{3}}(\bar{n} \bar{b})_{1}^{3}\right\rangle_{0} \\
\left|\{n s\}_{0}^{6}(\bar{n} \bar{b})_{0}^{\overline{6}}\right\rangle_{0}\end{array}$ & $\left(\begin{array}{cc}6516 & 140 \\
140 & 6628\end{array}\right)$ & {$\left[\begin{array}{l}6421 \\
6723\end{array}\right]$} & {$\left[\begin{array}{c}(-0.828,0.560) \\
(-0.560,-0.828)\end{array}\right]$} \\
\hline
\end{tabular}


TABLE III. (Continued)

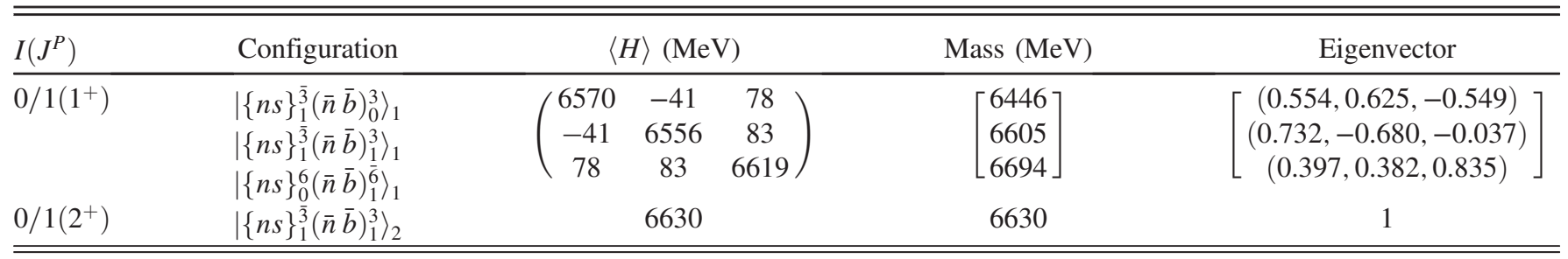

overlap with the excited charmed and bottom mesons. From the Review of Particle Physics [91], there exist several higher charmed states, which may correspond to the $n n \bar{n} \bar{c}$ tetraquark states. However, these observed resonances can be well described under the conventional interpretations. In fact, the physical resonances may be the admixtures of the conventional mesons and tetraquarks, which disturbs our understanding. The more efficient way is to hunt for the flavor-exotic states $u u \bar{d} \bar{c}, d d \bar{u} \bar{c}, u u \bar{d} \bar{b}$, and $d d \bar{u} \bar{b}$, which cannot mix with conventional mesons. With a large phase space, these flavor-exotic states and their antiparticles can easily decay into the conventional charmed or bottom mesons by emitting one or more pions, which can be searched for in future experiments.

There exist several types of flavor content for the tetraquark states including one strange quark, and the calculated mass spectra are shown in Table III. Given the $D^{-} K^{+}$decay mode, the newly observed $X_{0}(2900)$ and $X_{1}(2900)$ should belong to the $u d \bar{s} \bar{c}$ states, and their isospins can be either 0 or 1 . From Table III, it can be seen that the predicted masses of $0\left(0^{+}\right)$states are 2765 and $3152 \mathrm{MeV}$, where the large splitting arises from the significant mixing scheme of pure $|\overline{3} 3\rangle$ and $|6 \overline{6}\rangle$ states. Also, the mass of the lowest $1\left(0^{+}\right) n n \bar{s} \bar{c}$ state is $3065 \mathrm{MeV}$, which is larger than the experimental data. Our results disfavor the observed $X_{0}(2900)$ as a compact $u d \bar{s} \bar{c}$ tetraquark. Since the parity of $X_{1}(2900)$ is negative, it has one orbital excitation at least. From our calculations of $S$-wave states, the $P$-wave $n n \bar{s} \bar{c}$ states should have rather large masses, which excludes the assignment of $X_{1}(2900)$ as a $n n \bar{s} \bar{c}$ compact tetraquark state. Other interpretations, such as molecules and kinematic effects, are possible for these two states.
In Ref. [82] the authors adopted the color-magnetic interaction model to obtain four $0^{+} c s \bar{u} \bar{d}$ states with masses of $2320,2607,2850$, and $3129 \mathrm{MeV}$, respectively. It seems that the $X_{0}(2900)$ may be assigned as a higher $0^{+}$compact tetraquark through its mass, but the predicted decay width is about $10 \mathrm{MeV}$, which is significantly smaller than the experimental data. In Ref. [85] the lowest $0^{+} u d \bar{s} \bar{c}$ state was estimated to be $2754 \mathrm{MeV}$ in the baryonic-quark picture or $2863 \mathrm{MeV}$ in the string-junction picture within the simple quark model, where the $X_{0}(2900)$ can be regarded as a compact tetraquark. The color-magnetic interaction model and simple quark model also disfavor the $X_{1}(2900)$ as a compact tetraquark state, which is consistent with our calculations. It should be stressed that the differences among all of these works arise from the different choices of configurations, interactions, and parameters. In Ref. [85] only the diquark configurations under the $|\overline{3} 3\rangle$ representation were employed, while the four-body configurations were introduced in Ref. [82] and are used in our present work. Also, only the color-magnetic interactions are involved in Ref. [82], while the Coulomb potential, confining potential, spin-spin interactions, and the relativistic corrections are all considered in the present work. Moreover, the parameters in all of these mentioned models are quite different and based on different inputs. In our present work, the mixing effect between $\left|[n n]_{0}^{\overline{3}}(\bar{s} \bar{c})_{0}^{3}\right\rangle_{0}$ and $\left|[n n]_{1}^{6}(\bar{s} \bar{c})_{1}^{\overline{6}}\right\rangle_{0}$ is significant due to the large nondiagonal matrix elements. Even if only the pure $\left|[n n]_{0}^{\overline{3}}(\bar{s} \bar{c})_{0}^{3}\right\rangle_{0}$ configuration is considered here, we still cannot obtain the same results as Ref. [85] due to the different interactions and parameters. Finally, it is worth emphasizing that we adopt the parameters that describe the conventional mesons well and this unified treatment for mesons and tetraquarks is essential to obtain reliable mass

TABLE IV. The color proportions and root-mean-square radii of the lower $n n \bar{s} \bar{c}$ states. The expectations $\left\langle\boldsymbol{r}_{14}^{2}\right\rangle^{1 / 2}$, $\left\langle\boldsymbol{r}_{23}^{2}\right\rangle^{1 / 2}$, and $\left\langle\boldsymbol{r}^{\prime \prime 2}\right\rangle^{1 / 2}$ are equal to the values of $\left\langle\boldsymbol{r}_{24}^{2}\right\rangle^{1 / 2},\left\langle\boldsymbol{r}_{13}^{2}\right\rangle^{1 / 2}$, and $\left\langle\boldsymbol{r}^{2}\right\rangle^{1 / 2}$, respectively, which are omitted for simplicity. The masses and root-mean-square radii are in units of $\mathrm{MeV}$ and $\mathrm{fm}$, respectively.

\begin{tabular}{lccccccccc}
\hline \hline$I\left(J^{P}\right)$ & Mass & $|\overline{3} 3\rangle$ & $|6 \overline{6}\rangle$ & $\left\langle\boldsymbol{r}_{12}^{2}\right\rangle^{1 / 2}$ & $\left\langle\boldsymbol{r}_{34}^{2}\right\rangle^{1 / 2}$ & $\left\langle\boldsymbol{r}^{2}\right\rangle^{1 / 2}$ & $\left\langle\boldsymbol{r}_{13}^{2}\right\rangle^{1 / 2}$ & $\left\langle\boldsymbol{r}_{24}^{2}\right\rangle^{1 / 2}$ & $\left\langle\boldsymbol{r}^{\prime 2}\right\rangle^{1 / 2}$ \\
\hline $0\left(0^{+}\right)$ & 2765 & $45.8 \%$ & $54.2 \%$ & 0.524 & 0.480 & 0.351 & 0.581 & 0.449 & 0.367 \\
$0\left(1^{+}\right)$ & 2964 & $68.4 \%$ & $31.6 \%$ & 0.533 & 0.520 & 0.388 & 0.627 & 0.482 & 0.395 \\
$0\left(2^{+}\right)$ & 3316 & $0 \%$ & $100 \%$ & 0.688 & 0.640 & 0.344 & 0.704 & 0.504 & 0.483 \\
$1\left(0^{+}\right)$ & 3065 & $67.7 \%$ & $32.3 \%$ & 0.616 & 0.529 & 0.389 & 0.650 & 0.507 & 0.407 \\
$1\left(1^{+}\right)$ & 3130 & $70.0 \%$ & $30.0 \%$ & 0.622 & 0.521 & 0.407 & 0.659 & 0.523 & 0.404 \\
$1\left(2^{+}\right)$ & 3302 & $100 \%$ & $0 \%$ & 0.603 & 0.518 & 0.457 & 0.685 & 0.558 & 0.403 \\
\hline \hline
\end{tabular}




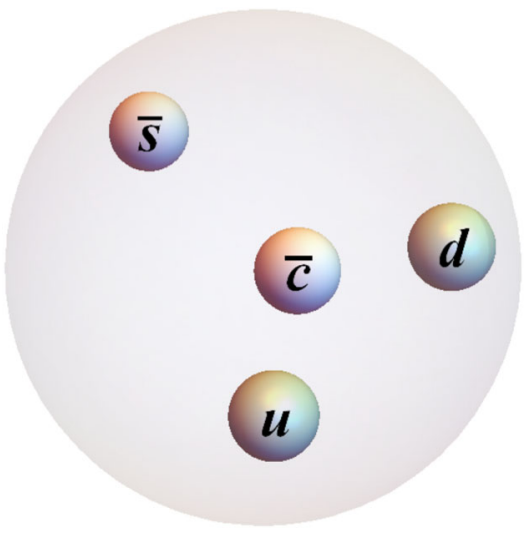

FIG. 2. Sketch of lower $u d \bar{s} \bar{c}$ states.

spectra of open charm and bottom tetraquarks. Further investigations with various approaches are encouraged.

The predicted color proportions and root-mean-square radii of the lower $n n \bar{s} \bar{c}$ states are listed in Table IV. Our results show that these states have relatively small rootmean-square radii, which indicates that all of them have compact inner structures. The sketch of these $u d \bar{s} \bar{c}$ tetraquarks is also plotted in Fig. 2. It can be seen that the four quarks are separated from each other in a compact tetraquark, which is quite different with the diquarkantidiquark or loosely bound molecular picture.

The $n n \bar{s} \bar{b}$ states are the bottom partners of $n n \bar{s} \bar{c}$ states, and all of the predicted masses lie above the respective thresholds. In Ref. [76] the author proposed a possible stable $b s \bar{u} \bar{d}$ state, while the calculations under the potential model indicate that no stable diquark-antidiquark $b s \bar{u} \bar{d}$ state exists [78]. Also, the color-magnetic interaction model suggests that the lowest $0^{+}$and $1^{+}$compact $b s \bar{u} \bar{d}$ states should be near the relevant thresholds [82]. More theoretical and experimental efforts are needed to clarify this problem.

Unlike the $n n \bar{s} \bar{c}$ and $n n \bar{s} \bar{b}$ states, some of the $n s \bar{n} \bar{c}$ and $n s \bar{n} \bar{b}$ states can mix with the conventional charmed-strange and bottom-strange mesons. At the early stage, the investigations on $n s \bar{n} \bar{c}$ and $n s \bar{n} \bar{b}$ states mainly focused on the possible tetraquark interpretation of the $D_{s 0}^{*}(2317)$. Since the light quark pair can be created or annihilated easily, it is difficult to distinguish various explanations of $D_{s 0}^{*}(2317)$. Hence, searching for the flavor-exotic states $u s \bar{d} \bar{c}, d s \bar{u} \bar{c}$, $u s \bar{d} \bar{b}$, and $d s \bar{u} \bar{b}$ seems to be more worthwhile. After the observation of $X(5568)$, plenty of studies on the $n s \bar{n} \bar{b}$

TABLE V. Predicted mass spectra for the $n s \bar{s} \bar{c}, n s \bar{s} \bar{b}, s s \bar{n} \bar{c}$, and $s s \bar{n} \bar{b}$ systems.

\begin{tabular}{|c|c|c|c|c|}
\hline$I\left(J^{P}\right)$ & Configuration & $\langle H\rangle(\mathrm{MeV})$ & Mass $(\mathrm{MeV})$ & Eigenvector \\
\hline$\frac{1}{2}\left(0^{+}\right)$ & $\begin{array}{l}\left|[n s]_{0}^{\overline{3}}(\bar{s} \bar{c})_{0}^{3}\right\rangle_{0} \\
\left|[n s]_{1}^{6}(\bar{s} \bar{c})_{1}^{6}\right\rangle_{0}\end{array}$ & $\left(\begin{array}{cc}3175 & 168 \\
168 & 3104\end{array}\right)$ & {$\left[\begin{array}{l}2967 \\
3312\end{array}\right]$} & {$\left[\begin{array}{c}(-0.630,0.776) \\
(0.776,0.630)\end{array}\right]$} \\
\hline$\frac{1}{2}\left(1^{+}\right)$ & $\begin{array}{l}\left|[n s]_{0}^{\overline{3}}(\bar{s} \bar{c})_{1}^{3}\right\rangle_{1} \\
\left|[n s]_{1}^{6}(\bar{s} \bar{c})_{0}^{6}\right\rangle_{1} \\
\left|[n s]_{1}^{6}(\bar{s} \bar{c})_{1}^{6}\right\rangle_{1}\end{array}$ & $\left(\begin{array}{ccc}3227 & -80 & 36 \\
-80 & 3357 & 37 \\
36 & 37 & 3231\end{array}\right)$ & {$\left[\begin{array}{l}3156 \\
3261 \\
3397\end{array}\right]$} & {$\left[\begin{array}{c}(0.732,0.397,-0.553) \\
(0.548,0.139,0.825) \\
(-0.404,0.907,0.116)\end{array}\right]$} \\
\hline$\frac{1}{2}\left(2^{+}\right)$ & $\left|[n s]_{1}^{6}(\bar{s} \bar{c})_{1}^{6}\right\rangle_{2}$ & 3438 & 3438 & 1 \\
\hline$\frac{1}{2}\left(0^{+}\right)$ & $\begin{array}{l}\left|\{n s\}_{1}^{3}(\bar{s} \bar{c})_{1}^{3}\right\rangle_{0} \\
\left|\{n s\}_{0}^{6}(\bar{s} \bar{c})_{0}^{6}\right\rangle_{0}\end{array}$ & $\left(\begin{array}{cc}3317 & 138 \\
138 & 3406\end{array}\right)$ & {$\left[\begin{array}{l}3217 \\
3506\end{array}\right]$} & {$\left[\begin{array}{c}(-0.808,0.589) \\
(-0.589,-0.808)\end{array}\right]$} \\
\hline$\frac{1}{2}\left(1^{+}\right)$ & $\begin{array}{l}\left|\{n s\}_{1}^{\overline{3}}(\bar{s} \bar{c})_{0}^{3}\right\rangle_{1} \\
\left|\{n s\}_{1}^{3}(\bar{s} \bar{c})_{1}^{3}\right\rangle_{1} \\
\left|\{n s\}_{0}^{6}(\bar{s} \bar{c})_{1}^{\overline{6}}\right\rangle_{1}\end{array}$ & $\left(\begin{array}{ccc}3344 & 20 & 78 \\
20 & 3357 & -34 \\
78 & -34 & 3391\end{array}\right)$ & {$\left[\begin{array}{l}3271 \\
3370 \\
3451\end{array}\right]$} & {$\left[\begin{array}{l}(0.720,-0.389,-0.575) \\
(-0.417,-0.905,0.090) \\
(-0.555,0.175,-0.813)\end{array}\right]$} \\
\hline$\frac{1}{2}\left(2^{+}\right)$ & $\left|\{n s\}_{1}^{3}(\bar{s} \bar{c})_{1}^{3}\right\rangle_{2}$ & 3431 & 3431 & 1 \\
\hline$\frac{1}{2}\left(0^{+}\right)$ & $\begin{array}{l}\left|[n s]_{0}^{\overline{3}}(\bar{s} \bar{b})_{0}^{3}\right\rangle_{0} \\
\left|[n s]_{1}^{6}(\bar{s} \bar{b})_{1}^{\overline{6}}\right\rangle_{0}\end{array}$ & $\left(\begin{array}{cc}6511 & 127 \\
127 & 6520\end{array}\right)$ & {$\left[\begin{array}{l}6388 \\
6643\end{array}\right]$} & {$\left[\begin{array}{c}(-0.719,0.695) \\
(-0.695,-0.719)\end{array}\right]$} \\
\hline$\frac{1}{2}\left(1^{+}\right)$ & $\begin{array}{l}\left|[n s]_{0}^{3}(\bar{s} \bar{b})_{1}^{3}\right\rangle_{1} \\
\left|[n s]_{1}^{6}(\bar{s} \bar{b})_{0}^{6}\right\rangle_{1} \\
\left|[n s]_{1}^{6}(\bar{s} \bar{b})_{1}^{\overline{6}}\right\rangle_{1}\end{array}$ & $\left(\begin{array}{ccc}6532 & -63 & -62 \\
-63 & 6705 & -72 \\
-62 & -72 & 6614\end{array}\right)$ & {$\left[\begin{array}{l}6464 \\
6639 \\
6748\end{array}\right]$} & {$\left[\begin{array}{c}(-0.789,-0.357,-0.500) \\
(-0.598,0.261,0.758) \\
(0.141,-0.897,0.419)\end{array}\right]$} \\
\hline$\frac{1}{2}\left(2^{+}\right)$ & $\left|[n s]_{1}^{6}(\bar{s} \bar{b})_{1}^{\overline{6}}\right\rangle_{2}$ & 6772 & 6772 & 1 \\
\hline$\frac{1}{2}\left(0^{+}\right)$ & $\begin{array}{l}\left|\{n s\}_{1}^{\overline{3}}(\bar{s} \bar{b})_{1}^{3}\right\rangle_{0} \\
\left|\{n s\}_{0}^{6}(\bar{s} \bar{b})_{0}^{6}\right\rangle_{0}\end{array}$ & $\left(\begin{array}{cc}6638 & -108 \\
-108 & 6753\end{array}\right)$ & {$\left[\begin{array}{l}6573 \\
6818\end{array}\right]$} & {$\left[\begin{array}{c}(-0.858,-0.513) \\
(0.513,-0.858)\end{array}\right]$} \\
\hline$\frac{1}{2}\left(1^{+}\right)$ & $\begin{array}{l}\left|\{n s\}_{1}^{\overline{3}}(\bar{s} \bar{b})_{0}^{3}\right\rangle_{1} \\
\left|\{n s\}_{1}^{\overline{3}}(\bar{s} \bar{b})_{1}^{3}\right\rangle_{1} \\
\left|\{n s\}_{0}^{6}(\bar{s} \bar{b})_{1}^{\overline{6}}\right\rangle_{1}\end{array}$ & $\left(\begin{array}{ccc}6679 & -30 & -60 \\
-30 & 6669 & -57 \\
-60 & -57 & 6747\end{array}\right)$ & {$\left[\begin{array}{l}6598 \\
6704 \\
6793\end{array}\right]$} & {$\left[\begin{array}{c}(0.597,0.637,0.487) \\
(0.706,-0.706,0.057) \\
(-0.379,-0.310,0.872)\end{array}\right]$} \\
\hline
\end{tabular}


TABLE V. (Continued)

\begin{tabular}{|c|c|c|c|c|}
\hline$I\left(J^{P}\right)$ & Configuration & $\langle H\rangle(\mathrm{MeV})$ & Mass $(\mathrm{MeV})$ & Eigenvector \\
\hline$\overline{\frac{1}{2}\left(2^{+}\right)}$ & $\left|\{n s\}_{1}^{\overline{3}}(\bar{s} \bar{b})_{1}^{3}\right\rangle_{2}$ & 6728 & 6728 & 1 \\
\hline$\frac{1}{2}\left(0^{+}\right)$ & $\begin{array}{l}\left|\{s s\}_{1}^{\frac{1}{3}}(\bar{n} \bar{c})_{1}^{3}\right\rangle_{0} \\
\left|\{s s\}_{0}^{6}(\bar{n} \bar{c})_{0}^{6}\right\rangle_{0}\end{array}$ & $\left(\begin{array}{rr}3326 & -144 \\
-144 & 3413\end{array}\right)$ & {$\left[\begin{array}{l}3219 \\
3520\end{array}\right]$} & {$\left[\begin{array}{c}(-0.802,-0.570) \\
(0.570,-0.802)\end{array}\right]$} \\
\hline$\frac{1}{2}\left(1^{+}\right)$ & $\begin{array}{l}\left|\{s s\}_{1}^{\overline{3}}(\bar{n} \bar{c})_{0}^{3}\right\rangle_{1} \\
\left|\{s s\}_{1}^{3}(\bar{n} \bar{c})_{1}^{3}\right\rangle_{1} \\
\left|\{s s\}_{0}^{6}(\bar{n} \bar{c})_{1}^{\overline{6}}\right\rangle_{1}\end{array}$ & $\left(\begin{array}{ccc}3344 & -24 & 81 \\
-24 & 3368 & 44 \\
81 & 44 & 3392\end{array}\right)$ & {$\left[\begin{array}{l}3263 \\
3382 \\
3458\end{array}\right]$} & {$\left[\begin{array}{c}(0.705,0.404,-0.583) \\
(-0.475,0.880,0.035) \\
(0.527,0.252,0.812)\end{array}\right]$} \\
\hline$\frac{1}{2}\left(2^{+}\right)$ & $\left|\{s s\}_{1}^{\overline{3}}(\bar{n} \bar{c})_{1}^{3}\right\rangle_{2}$ & 3443 & 3443 & 1 \\
\hline$\frac{1}{2}\left(0^{+}\right)$ & $\begin{array}{l}\left|\{s s\}_{1}^{\overline{3}}(\bar{n} \bar{b})_{1}^{3}\right\rangle_{0} \\
\left|\{s s\}_{0}^{6}(\bar{n} \bar{b})_{0}^{\overline{6}}\right\rangle_{0}\end{array}$ & $\left(\begin{array}{cc}6631 & -120 \\
-120 & 6716\end{array}\right)$ & {$\left[\begin{array}{l}6547 \\
6800\end{array}\right]$} & {$\left[\begin{array}{c}(-0.816,-0.577) \\
(0.577,-0.816)\end{array}\right]$} \\
\hline$\frac{1}{2}\left(1^{+}\right)$ & $\begin{array}{l}\left|\{s s\}_{1}^{\overline{3}}(\bar{n} \bar{b})_{0}^{3}\right\rangle_{1} \\
\left|\{s s\}_{1}^{3}(\bar{n} \bar{b})_{1}^{3}\right\rangle_{1} \\
\left|\{s s\}_{0}^{6}(\bar{n} \bar{b})_{1}^{\overline{6}}\right\rangle_{1}\end{array}$ & $\left(\begin{array}{ccc}6674 & -34 & 67 \\
-34 & 6665 & 69 \\
67 & 69 & 6707\end{array}\right)$ & {$\left[\begin{array}{l}6569 \\
6704 \\
6773\end{array}\right]$} & {$\left[\begin{array}{c}(0.559,0.602,-0.571) \\
(0.715,-0.698,-0.035) \\
(0.419,0.388,0.821)\end{array}\right]$} \\
\hline$\frac{1}{2}\left(2^{+}\right)$ & $\left|\{s s\}_{1}^{\overline{3}}(\bar{n} \bar{b})_{1}^{3}\right\rangle_{2}$ & 6729 & 6729 & 1 \\
\hline
\end{tabular}

system have been performed. Our results for the $n s \bar{n} \bar{c}$ and $n s \bar{n} \bar{b}$ states are much higher than the masses of $D_{s 0}^{*}(2317)$ and $X(5568)$, which exclude their tetraquark interpretations.

The tetraquarks $n n \bar{s} \bar{c}$ and $n n \bar{s} \bar{b}$ can decay into the $\bar{D}^{(*)} K^{(*)}$ and $B^{(*)} K^{(*)}$ final states, respectively, via the fallapart mechanism. For the $n s \bar{n} \bar{c}$ and $n s \bar{n} \bar{b}$ states, the possible decay channels are $\bar{D}^{(*)} \bar{K}^{(*)}, D_{s}^{(*)-} \pi, B^{(*)} \bar{K}^{(*)}$, and $B_{s}^{(*)} \pi$. Certainly, the antiparticles of these tetraquarks decay into similar final states under a charge conjugation transformation. We hope that our results can provide helpful information for hunting these flavor-exotic tetraquarks in future $\mathrm{LHCb}$ and Belle II experiments.

The masses of tetraquark states including two strange quarks are listed in Table V. For the $n s \bar{s} \bar{c}$ and $n s \bar{s} \bar{b}$ states, the predicted masses are much higher than the relevant thresholds, which may fall apart easily. Also, these states can mix with the conventional charmed and bottom mesons, and be hardly picked out from the excited mesons. The more interesting systems are the $s s \bar{n} \bar{c}$ and $s s \bar{n} \bar{b}$ states, which are totally flavor exotic. They and their antiparticles can be searched for in the $D_{s}^{(*)-} \bar{K}^{(*)}, D_{s}^{(*)+} K^{(*)}, B_{s}^{(*)} \bar{K}^{(*)}$, and $\bar{B}_{s}^{(*)} K^{(*)}$ final states by future experiments.

The calculated masses of $s s \bar{s} \bar{c}$ and $s s \bar{s} \bar{b}$ systems are presented in Table VI, which lie above 3300 and $6600 \mathrm{MeV}$, respectively. These states can mix with the conventional charmed-strange and bottom-strange mesons via strange-quark pair annihilation. Current experiments have not investigated these energy regions, and future experimental searches can test our calculations.

Finally, we plot the full mass spectra of open charm and bottom tetraquarks in Fig. 3. It can be seen that the spectra for various systems show quite similar patterns, which indicates that the approximate light flavor SU(3) symmetry

TABLE VI. Predicted mass spectra for the $s s \bar{s} \bar{c}$ and $s s \bar{s} \bar{b}$ systems.

\begin{tabular}{|c|c|c|c|c|}
\hline$I\left(J^{P}\right)$ & Configuration & $\langle H\rangle(\mathrm{MeV})$ & Mass $(\mathrm{MeV})$ & Eigenvector \\
\hline $0\left(0^{+}\right)$ & $\begin{array}{l}\left|\{s s\}_{1}^{\overline{3}}(\bar{s} \bar{c})_{1}^{3}\right\rangle_{0} \\
\left|\{s s\}_{0}^{6}(\bar{s} \bar{c})_{0}^{6}\right\rangle_{0}\end{array}$ & $\left(\begin{array}{cc}3429 & 126 \\
126 & 3494\end{array}\right)$ & {$\left[\begin{array}{l}3331 \\
3592\end{array}\right]$} & {$\left[\begin{array}{c}(-0.790,0.613) \\
(-0.613,-0.790)\end{array}\right]$} \\
\hline $0\left(1^{+}\right)$ & $\begin{array}{l}\left|\{s s\}_{1}^{\overline{3}}(\bar{s} \bar{c})_{0}^{3}\right\rangle_{1} \\
\left|\{s s\}_{1}^{3}(\bar{s} \bar{c})_{1}^{3}\right\rangle_{1} \\
\left|\{s s\}_{0}^{6}(\bar{s} \bar{c})_{1}^{\overline{6}}\right\rangle_{1}\end{array}$ & $\left(\begin{array}{ccc}3448 & -17 & 71 \\
-17 & 3466 & 29 \\
71 & 29 & 3479\end{array}\right)$ & {$\left[\begin{array}{l}3379 \\
3475 \\
3539\end{array}\right]$} & {$\left[\begin{array}{c}(0.716,0.339,-0.610) \\
(-0.374,0.924,0.075) \\
(0.590,0.174,0.789)\end{array}\right]$} \\
\hline $0\left(2^{+}\right)$ & $\left|\{s s\}_{1}^{\overline{3}}(\bar{s} \bar{c})_{1}^{3}\right\rangle_{2}$ & 3533 & 3533 & 1 \\
\hline $0\left(0^{+}\right)$ & $\begin{array}{l}\left|\{s s\}_{1}^{\overline{3}}(\bar{s} \bar{b})_{1}^{3}\right\rangle_{0} \\
\left|\{s s\}_{0}^{6}(\bar{s} \bar{b})_{0}^{6}\right\rangle_{0}\end{array}$ & $\left(\begin{array}{cc}6745 & 98 \\
98 & 6835\end{array}\right)$ & {$\left[\begin{array}{l}6682 \\
6898\end{array}\right]$} & {$\left[\begin{array}{c}(-0.843,0.538) \\
(-0.538,-0.843)\end{array}\right]$} \\
\hline $0\left(1^{+}\right)$ & $\begin{array}{l}\left|\{s s\}_{1}^{\overline{3}}(\bar{s} \bar{b})_{0}^{3}\right\rangle_{1} \\
\left|\{s s\}_{1}^{3}(\bar{s} \bar{b})_{1}^{3}\right\rangle_{1} \\
\left|\{s s\}_{0}^{6}(\bar{s} \bar{b})_{1}^{\overline{6}}\right\rangle_{1}\end{array}$ & $\left(\begin{array}{ccc}6779 & 26 & -55 \\
26 & 6773 & 50 \\
-55 & 50 & 6828\end{array}\right)$ & {$\left[\begin{array}{l}6705 \\
6802 \\
6874\end{array}\right]$} & $\begin{array}{c}(0.598,-0.612,0.517) \\
(-0.690,-0.722,-0.057) \\
(-0.408,0.322,0.854)\end{array}$ \\
\hline $0\left(2^{+}\right)$ & $\left|\{s s\}_{1}^{\overline{3}}(\bar{s} \bar{b})_{1}^{3}\right\rangle_{2}$ & 6826 & 6826 & 1 \\
\hline
\end{tabular}



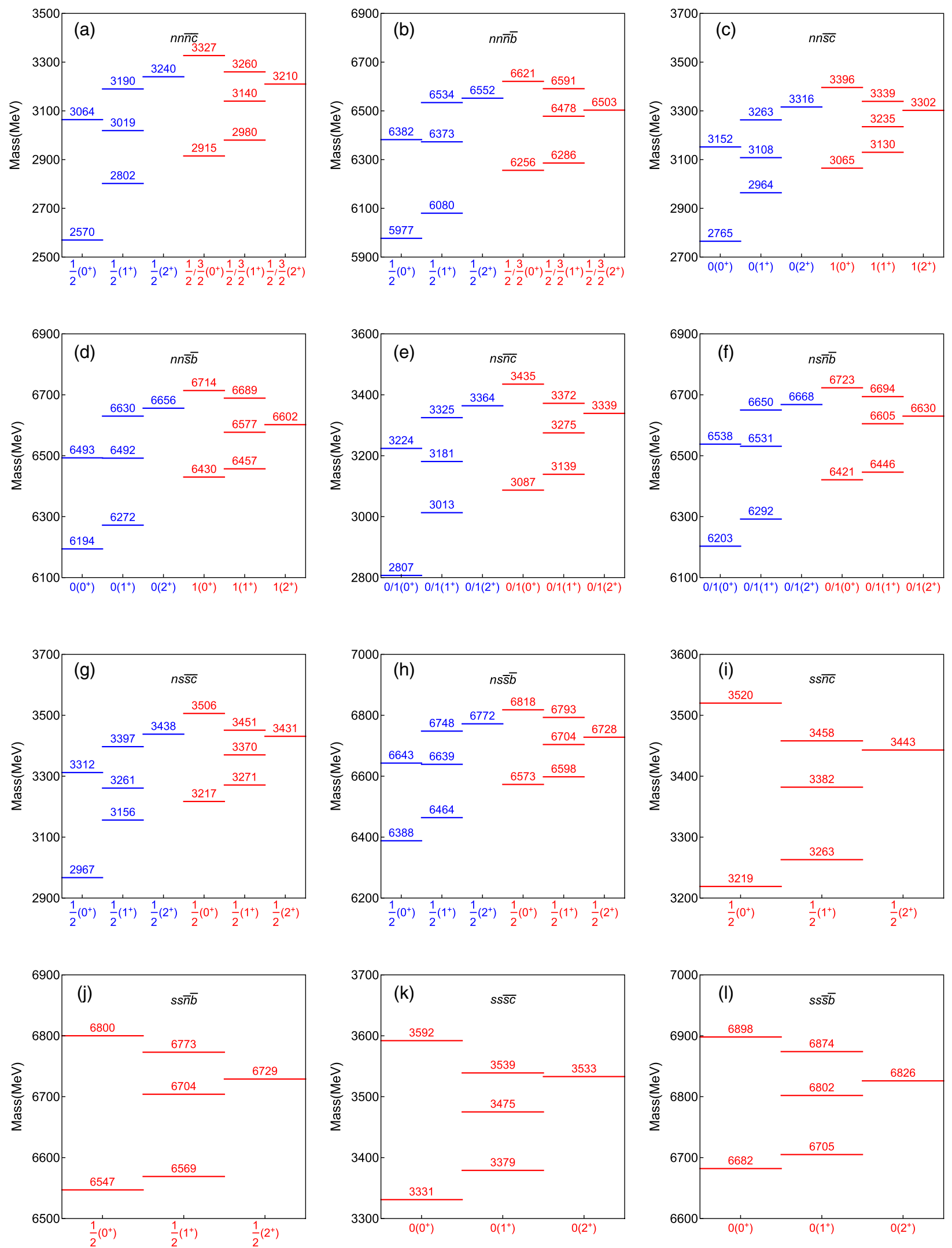

FIG. 3. The predicted mass spectra of open charm and bottom tetraquarks. (a)-(1) correspond to the mass spectra of $n n \bar{n} \bar{c}, n n \bar{n} \bar{b}, n n \bar{s} \bar{c}, n n \bar{s} \bar{b}, n s \bar{n} \bar{c}, n s \bar{n} \bar{b}, n s \bar{s} \bar{c}, n s \bar{s} \bar{b}, s s \bar{n} \bar{c}, s s \bar{n} \bar{b}, s s \bar{s} \bar{c}$, and $s s \bar{s} \bar{b}$, states, respectively. 
and heavy quark symmetry are preserved well for the ground states of singly heavy tetraquarks. These two symmetries have been successful for the traditional hadrons, which will also provide a powerful tool for us to investigate the singly heavy tetraquarks. Compared with the extensive studies of conventional heavy-light mesons and singly heavy baryons, the studies on singly heavy tetraquarks are lacking. More theoretical and experimental efforts are encouraged to increase our understanding of these systems.

\section{SUMMARY}

In this work we systematically investigated the mass spectra of open charm and bottom tetraquarks $q q \bar{q} \bar{Q}$ within an extended relativized quark model. By using the variational method, the four-body relativized Hamiltonian including the Coulomb potential, confining potential, spin-spin interactions, and relativistic corrections were solved. The predicted masses of four $0^{+} u d \bar{s} \bar{c}$ states are $2765,3065,3152$, and $3396 \mathrm{MeV}$, which disfavors the assignment of $X_{0}(2900)$ as a compact tetraquark.

The $S$-wave mass spectra of open charm and bottom tetraquarks show quite similar patterns, which preserves the light flavor SU(3) symmetry and heavy quark symmetry well. Besides the mass spectra, the possible decay modes were also discussed. Our results suggest that future experiments can search for the flavor-exotic states $n n \bar{s} \bar{c}, n n \bar{s} \bar{b}$, $s \operatorname{s} \bar{c}$, and $s \operatorname{s} \bar{b}$ in the heavy-light meson plus kaon final states. More theoretical and experimental efforts are needed to investigate these singly heavy tetraquarks.

\section{ACKNOWLEDGMENTS}

We would like to thank Xian-Hui Zhong for valuable discussions. This project is supported by the National Natural Science Foundation of China under Grants No. 11705056, No. 11775050, No. 11947224, No. 11975245, and No. U1832173, by the fund provided to the Sino-German CRC 110 "Symmetries and the Emergenceof Structure in QCD" project by the NSFC under Grant No. 11621131001, and by the Key Research Program of Frontier Sciences, CAS, Grant No. Y7292610K1.

\section{APPENDIX: THE RELATIVIZED POTENTIALS}

In the present work, we only concentrate on the $S$-wave ground states and do not include the spin-orbit and tensor interactions. The potential $V_{i j}^{\text {oge }}$ can be expressed as

$$
V_{i j}^{\mathrm{oge}}=\beta_{i j}^{1 / 2} \tilde{G}\left(r_{i j}\right) \beta_{i j}^{1 / 2}+\delta_{i j}^{1 / 2+\epsilon_{c}} \frac{2 S_{i} \cdot S_{j}}{3 m_{i} m_{j}} \nabla^{2} \tilde{G}\left(r_{i j}\right) \delta_{i j}^{1 / 2+\epsilon_{c}},
$$

with

$$
\beta_{i j}=1+\frac{p_{i j}^{2}}{\left(p_{i j}^{2}+m_{i}^{2}\right)^{1 / 2}\left(p_{i j}^{2}+m_{j}^{2}\right)^{1 / 2}}
$$

and

$$
\delta_{i j}=\frac{m_{i} m_{j}}{\left(p_{i j}^{2}+m_{i}^{2}\right)^{1 / 2}\left(p_{i j}^{2}+m_{j}^{2}\right)^{1 / 2}} .
$$

$p_{i j}$ is the magnitude of the momentum of either of the quarks in the center-of-mass frame of the $i j$ quark subsystem, and $\epsilon_{c}$ is a free parameter reflecting the momentum dependence. The smeared Coulomb potential $\tilde{G}\left(r_{i j}\right)$ is

$$
\tilde{G}\left(r_{i j}\right)=\boldsymbol{F}_{i} \cdot \boldsymbol{F}_{j} \sum_{k=1}^{3} \frac{\alpha_{k}}{r_{i j}} \operatorname{erf}\left(\tau_{k i j} r_{i j}\right),
$$

with

$$
\frac{1}{\tau_{k i j}^{2}}=\frac{1}{\gamma_{k}^{2}}+\frac{1}{\sigma_{i j}^{2}}
$$

and

$\sigma_{i j}^{2}=\sigma_{0}^{2}\left[\frac{1}{2}+\frac{1}{2}\left(\frac{4 m_{i} m_{j}}{\left(m_{i}+m_{j}\right)^{2}}\right)^{4}\right]+s^{2}\left(\frac{2 m_{i} m_{j}}{m_{i}+m_{j}}\right)^{2}$.

$\boldsymbol{F}_{i} \cdot \boldsymbol{F}_{j}$ stands for the color matrix and reads

$$
\boldsymbol{F}_{i}= \begin{cases}\frac{\lambda_{i}}{2} & \text { for quarks } \\ -\frac{\lambda_{i}^{*}}{2} & \text { for antiquarks. }\end{cases}
$$

Similarly, the confining interaction $V_{i j}^{\text {conf }}$ can be expressed as

$$
\begin{aligned}
V_{i j}^{\mathrm{conf}}= & -\frac{3}{4} \boldsymbol{F}_{i} \cdot \boldsymbol{F}_{j} \\
& \times\left\{b r\left[\frac{e^{-\sigma_{i j}^{2} r^{2}}}{\sqrt{\pi} \sigma_{i j} r}+\left(1+\frac{1}{2 \sigma_{i j}^{2} r^{2}}\right) \operatorname{erf}\left(\sigma_{i j} r\right)\right]+c\right\} .
\end{aligned}
$$

All of the parameters used here are taken from the original reference [89] and collected in Table VII for convenience.

TABLE VII. Relevant parameters.

\begin{tabular}{lcccc}
\hline \hline$m_{u} / m_{d}(\mathrm{MeV})$ & $m_{s}(\mathrm{MeV})$ & $m_{c}(\mathrm{MeV})$ & $m_{b}(\mathrm{MeV})$ & $\alpha_{1}$ \\
220 & 419 & 1628 & 4977 & 0.25 \\
\hline$\alpha_{2}$ & $\alpha_{3}$ & $\gamma_{1}(\mathrm{GeV})$ & $\gamma_{2}(\mathrm{GeV})$ & $\gamma_{3}(\mathrm{GeV})$ \\
0.15 & 0.20 & $1 / 2$ & $\sqrt{10} / 2$ & $\sqrt{1000} / 2$ \\
\hline$b\left(\mathrm{GeV}^{2}\right)$ & $c(\mathrm{MeV})$ & $\sigma_{0}(\mathrm{GeV})$ & $s$ & $\epsilon_{c}$ \\
0.18 & -253 & 1.80 & 1.55 & -0.168 \\
\hline \hline
\end{tabular}


[1] E. Klempt and A. Zaitsev, Glueballs, hybrids, multiquarks. Experimental facts versus QCD inspired concepts, Phys. Rep. 454, 1 (2007).

[2] N. Brambilla et al., Heavy Quarkonium: Progress, puzzles, and opportunities, Eur. Phys. J. C 71, 1534 (2011).

[3] A. Hosaka, T. Iijima, K. Miyabayashi, Y. Sakai, and S. Yasui, Exotic hadrons with heavy flavors: X, Y, Z, and related states, Prog. Theor. Exp. Phys. (2016), 062C01.

[4] H. X. Chen, W. Chen, X. Liu, and S. L. Zhu, The hiddencharm pentaquark and tetraquark states, Phys. Rep. 639, 1 (2016).

[5] R. F. Lebed, R. E. Mitchell, and E. S. Swanson, Heavyquark QCD exotica, Prog. Part. Nucl. Phys. 93, 143 (2017).

[6] A. Esposito, A. Pilloni, and A. D. Polosa, Multiquark resonances, Phys. Rep. 668, 1 (2017).

[7] Y. Dong, A. Faessler, and V. E. Lyubovitskij, Description of heavy exotic resonances as molecular states using phenomenological Lagrangians, Prog. Part. Nucl. Phys. 94, 282 (2017).

[8] A. Ali, J. S. Lange, and S. Stone, Exotics: Heavy pentaquarks and tetraquarks, Prog. Part. Nucl. Phys. 97, 123 (2017).

[9] F. K. Guo, C. Hanhart, U. G. Meißner, Q. Wang, Q. Zhao, and B.S. Zou, Hadronic molecules, Rev. Mod. Phys. 90, 015004 (2018).

[10] S. L. Olsen, T. Skwarnicki, and D. Zieminska, Nonstandard heavy mesons and baryons: Experimental evidence, Rev. Mod. Phys. 90, 015003 (2018).

[11] M. Karliner, J. L. Rosner, and T. Skwarnicki, Multiquark states, Annu. Rev. Nucl. Part. Sci. 68, 17 (2018).

[12] Y. R. Liu, H. X. Chen, W. Chen, X. Liu, and S. L. Zhu, Pentaquark and tetraquark states, Prog. Part. Nucl. Phys. 107, 237 (2019).

[13] N. Brambilla, S. Eidelman, C. Hanhart, A. Nefediev, C. P. Shen, C. E. Thomas, A. Vairo, and C.Z. Yuan, The $X Y Z$ states: Experimental and theoretical status and perspectives, Phys. Rep. 873, 1 (2020).

[14] J. M. Richard, A. Valcarce, and J. Vijande, Hall-post inequalities: Review and application to molecules and tetraquarks, Ann. Phys. (Amsterdam) 412, 168009 (2020).

[15] S. Choi et al. (Belle Collaboration), Observation of a Resonance-Like Structure in the $\pi^{ \pm} \psi^{\prime}$ Mass Distribution in Exclusive $B \rightarrow K \pi^{ \pm} \psi^{\prime}$ Decays, Phys. Rev. Lett. 100, 142001 (2008).

[16] R. Aaij et al. (LHCb Collaboration), Observation of the Resonant Character of the $Z(4430)^{-}$State, Phys. Rev. Lett. 112, 222002 (2014).

[17] A. Bondar et al. (Belle Collaboration), Observation of Two Charged Bottomonium-Like Resonances in $\Upsilon(5 S)$ Decays, Phys. Rev. Lett. 108, 122001 (2012).

[18] M. Ablikim et al. (BESIII Collaboration), Observation of a Charged Charmoniumlike Structure in $e^{+} e^{-} \rightarrow \pi^{+} \pi^{-} J / \psi$ at $\sqrt{s}=4.26 \mathrm{GeV}$, Phys. Rev. Lett. 110, 252001 (2013).

[19] Z. Liu et al. (Belle Collaboration), Study of $e^{+} e^{-} \rightarrow$ $\pi^{+} \pi^{-} J / \psi$ and Observation of a Charged Charmoniumlike State at Belle, Phys. Rev. Lett. 110, 252002 (2013).

[20] R. Aaij et al. (LHCb Collaboration), Observation of structure in the $J / \psi$-pair mass spectrum, arXiv:2006 .16957 .
[21] R. Aaij et al. (LHCb Collaboration), Observation of $J / \psi p$ Resonances Consistent with Pentaquark States in $\Lambda_{b}^{0} \rightarrow$ $J / \psi K^{-} p$ Decays, Phys. Rev. Lett. 115, 072001 (2015).

[22] R. Aaij et al. (LHCb Collaboration), Observation of a Narrow Pentaquark State, $P_{c}(4312)^{+}$, and of Two-Peak Structure of the $P_{c}(4450)^{+}$, Phys. Rev. Lett. 122, 222001 (2019).

[23] V. Abazov et al. (D0 Collaboration), Evidence for a $B_{s}^{0} \pi^{ \pm}$ State, Phys. Rev. Lett. 117, 022003 (2016).

[24] R. Aaij et al. (LHCb Collaboration), Search for Structure in the $B_{s}^{0} \pi^{ \pm}$Invariant Mass Spectrum, Phys. Rev. Lett. 117, 152003 (2016).

[25] A. M. Sirunyan et al. (CMS Collaboration), Search for the $X(5568)$ State Decaying into $\mathrm{B}_{\mathrm{s}}^{0} \pi^{ \pm}$in Proton-Proton Collisions at $\sqrt{s}=8 \mathrm{TeV}$, Phys. Rev. Lett. 120, 202005 (2018).

[26] T. Aaltonen et al. (CDF Collaboration), A Search for the Exotic Meson $X(5568)$ with the Collider Detector at Fermilab, Phys. Rev. Lett. 120, 202006 (2018).

[27] M. Aaboud et al. (ATLAS Collaboration), Search for a Structure in the $B_{s}^{0} \pi^{ \pm}$Invariant Mass Spectrum with the ATLAS Experiment, Phys. Rev. Lett. 120, 202007 (2018).

[28] V. M. Abazov et al. (D0 Collaboration), Study of the $X^{ \pm}(5568)$ state with semileptonic decays of the $B_{s}^{0}$ meson, Phys. Rev. D 97, 092004 (2018).

[29] M. E. Bracco, A. Lozea, R. D. Matheus, F. S. Navarra, and M. Nielsen, Disentangling two- and four-quark state pictures of the charmed scalar mesons, Phys. Lett. B 624, 217 (2005).

[30] L. Maiani, F. Piccinini, A. D. Polosa, and V. Riquer, Diquark-antidiquarks with hidden or open charm and the nature of $X(3872)$, Phys. Rev. D 71, 014028 (2005).

[31] J. Vijande, F. Fernandez, and A. Valcarce, Open-charm meson spectroscopy, Phys. Rev. D 73, 034002 (2006); Erratum, Phys. Rev. D 74, 059903 (2006).

[32] M. V. Carlucci, F. Giannuzzi, G. Nardulli, M. Pellicoro, and S. Stramaglia, AdS-QCD quark-antiquark potential, meson spectrum and tetraquarks, Eur. Phys. J. C 57, 569 (2008).

[33] H.X. Zhang, W. L. Wang, Y.-B. Dai, and Z. Y. Zhang, Chiral SU(3) quark model study of tetraquark states: $\operatorname{cn} \bar{n} \bar{s} / \operatorname{cs} \bar{s} \bar{s}$, Commun. Theor. Phys. 49, 414 (2008).

[34] S. M. Gerasyuta and V. I. Kochkin, Tetraquarks with charm in coupled-channel formalism, Phys. Rev. D 78, 116004 (2008).

[35] D. Ebert, R. N. Faustov, and V. O. Galkin, Masses of tetraquarks with open charm and bottom, Phys. Lett. B 696, 241 (2011).

[36] S. S. Agaev, K. Azizi, and H. Sundu, Mass and decay constant of the newly observed exotic $X(5568)$ state, Phys. Rev. D 93, 074024 (2016).

[37] W. Wang and R. Zhu, Can $X(5568)$ be a tetraquark state?, Chin. Phys. C 40, 093101 (2016).

[38] Z. G. Wang, Analysis of the $X(5568)$ as scalar tetraquark state in the diquark-antidiquark model with QCD sum rules, Commun. Theor. Phys. 66, 335 (2016).

[39] C. M. Zanetti, M. Nielsen, and K. P. Khemchandani, QCD sum rule study of a charged bottom-strange scalar meson, Phys. Rev. D 93, 096011 (2016).

[40] W. Chen, H. X. Chen, X. Liu, T. G. Steele, and S. L. Zhu, Decoding the $X(5568)$ as a Fully Open-Flavor $s u \bar{b} \bar{d}$ Tetraquark State, Phys. Rev. Lett. 117, 022002 (2016). 
[41] S. S. Agaev, K. Azizi, and H. Sundu, Width of the exotic $X_{b}(5568)$ state through its strong decay to $B_{s}^{0} \pi^{+}$, Phys. Rev. D 93, 114007 (2016).

[42] Y. R. Liu, X. Liu, and S. L. Zhu, $X(5568)$ and its partner states, Phys. Rev. D 93, 074023 (2016).

[43] J. M. Dias, K. P. Khemchandani, A. Martinez Torres, M. Nielsen, and C. M. Zanetti, A QCD sum rule calculation of the $X^{ \pm}(5568) \rightarrow B_{s}^{0} \pi^{ \pm}$decay width, Phys. Lett. B 758, 235 (2016).

[44] Z. G. Wang, Analysis of the strong decay $X(5568) \rightarrow B_{s}^{0} \pi^{+}$ with QCD sum rules, Eur. Phys. J. C 76, 279 (2016).

[45] F. Stancu, $X(5568)$ as a $s u \bar{d} \bar{b}$ tetraquark in a simple quark model, J. Phys. G 43, 105001 (2016).

[46] L. Tang and C. F. Qiao, Tetraquark states with open flavors, Eur. Phys. J. C 76, 558 (2016).

[47] A. Ali, L. Maiani, A. D. Polosa, and V. Riquer, $B_{c}^{ \pm}$decays into tetraquarks, Phys. Rev. D 94, 034036 (2016).

[48] S. S. Agaev, K. Azizi, and H. Sundu, Application of the QCD light cone sum rule to tetraquarks: The strong vertices $X_{b} X_{b} \rho$ and $X_{c} X_{c} \rho$, Phys. Rev. D 93, 114036 (2016).

[49] F. Goerke, T. Gutsche, M. A. Ivanov, J. G. Korner, V. E. Lyubovitskij, and P. Santorelli, Four-quark structure of $Z_{c}(3900), Z(4430)$ and $X_{b}(5568)$ states, Phys. Rev. D 94, 094017 (2016).

[50] S. S. Agaev, K. Azizi, B. Barsbay, and H. Sundu, Resonance $X(5568)$ as an exotic axial-vector state, Eur. Phys. J. A 53, 11 (2017).

[51] A. K. Agamaliev, T. M. Aliev, and M. Savc, Magnetic moment of $X_{Q}$ state with $J^{P C}=1^{+ \pm}$in light cone QCD sum rules, Phys. Rev. D 95, 036015 (2017).

[52] Q. F. Lü and Y. B. Dong, Masses of open charm and bottom tetraquark states in a relativized quark model, Phys. Rev. D 94, 094041 (2016).

[53] W. Chen, H. X. Chen, X. Liu, T. G. Steele, and S. L. Zhu, Open-flavor charm and bottom $s q \bar{q} \bar{Q}$ and $q q \bar{q} \bar{Q}$ tetraquark states, Phys. Rev. D 95, 114005 (2017).

[54] J. R. Zhang, J. L. Zou, and J. Y. Wu, $0^{+}$tetraquark states from improved QCD sum rules: delving into $X(5568)$, Chin. Phys. C 42, 043101 (2018).

[55] J. Y. Sng, A. Trkan, and E. Veli Veliev, In the pursuit of $X(5568)$ and its charmed partner, Acta Phys. Pol. B 50, 1501 (2019).

[56] C. J. Xiao and D. Y. Chen, Possible $B^{(*)} \bar{K}$ hadronic molecule state, Eur. Phys. J. A 53, 127 (2017).

[57] S. S. Agaev, K. Azizi, and H. Sundu, Exploring X(5568) as a meson molecule, Eur. Phys. J. Plus 131, 351 (2016).

[58] M. Albaladejo, J. Nieves, E. Oset, Z. F. Sun, and X. Liu, Can $X(5568)$ be described as a $B_{s} \pi, B \bar{K}$ resonant state?, Phys. Lett. B 757, 515 (2016).

[59] X. Chen and J. Ping, Is the exotic $X(5568)$ a bound state? Eur. Phys. J. C 76, 351 (2016).

[60] X. W. Kang and J. A. Oller, $P$-wave coupled-channel scattering of $B_{s} \pi, B_{s}^{*} \pi, B \bar{K}, B^{*} \bar{K}$ and the puzzling $X(5568)$, Phys. Rev. D 94, 054010 (2016).

[61] C. B. Lang, D. Mohler, and S. Prelovsek, $B_{s} \pi^{+}$scattering and search for $X(5568)$ with lattice QCD, Phys. Rev. D 94, 074509 (2016).

[62] R. Chen and X. Liu, Is the newly reported $X(5568)$ a $B \bar{K}$ molecular state? Phys. Rev. D 94, 034006 (2016).
[63] J. X. Lu, X. L. Ren, and L. S. Geng, $B_{s} \pi B \bar{K}$ interactions in finite volume and $X(5568)$, Eur. Phys. J. C 77, 94 (2017).

[64] B. X. Sun, F. Y. Dong, and J. L. Pang, Study of $X(5568)$ in a unitary coupled-channel approximation of $B \bar{K}$ and $B_{s} \pi$, Chin. Phys. C 41, 074104 (2017).

[65] H. W. Ke, L. Gao, and X. Q. Li, The possible $B \pi$ molecular state and its radiative decay, Eur. Phys. J. C 77, 285 (2017).

[66] X. H. Liu and G. Li, Could the observation of $X(5568)$ be a result of the near threshold rescattering effects? Eur. Phys. J. C 76, 455 (2016).

[67] Z. Yang, Q. Wang, and U. G. Meißner, Where does the $X(5568)$ structure come from?, Phys. Lett. B 767, 470 (2017).

[68] X. H. Liu and U. G. Meißner, Generating a resonance-like structure in the reaction $B_{c} \rightarrow B_{s} \pi \pi$, Eur. Phys. J. C 77, 816 (2017).

[69] A. Esposito, A. Pilloni, and A. D. Polosa, Hybridized tetraquarks, Phys. Lett. B 758, 292 (2016).

[70] S. S. Agaev, K. Azizi, and H. Sundu, Charmed partner of the exotic $X(5568)$ state and its properties, Phys. Rev. D 93, 094006 (2016).

[71] X. G. He and P. Ko, Flavor SU (3) roperties of beauty tetraquark states with three different light quarks, Phys. Lett. B 761, 92 (2016).

[72] Y. Jin, S. Y. Li, and S. Q. Li, New $B_{s}^{0} \pi^{ \pm}$and $D_{s}^{ \pm} \pi^{ \pm}$states in high energy multiproduction process, Phys. Rev. D 94, 014023 (2016).

[73] T. J. Burns and E. S. Swanson, Interpreting the $X(5568)$, Phys. Lett. B 760, 627 (2016).

[74] F. K. Guo, U. G. Meißner, and B. S. Zou, How the $X(5568)$ challenges our understanding of QCD, Commun. Theor. Phys. 65, 593 (2016).

[75] X. G. He, W. Wang, and R. L. Zhu, Production of charmed tetraquarks from $B_{c}$ and $B$ decays, J. Phys. G 44, 014003 (2017).

[76] F. S. Yu, $b s \bar{u} \bar{d}$ : A promising detectable tetraquark, arXiv:1709.02571.

[77] K. Azizi and U. zdem, The electromagnetic multipole moments of the charged open-flavor $Z_{\bar{c} q}$ states, J. Phys. G 45, 055003 (2018).

[78] X. Chen and J. Ping, Looking for a $u d \bar{s} \bar{b}$ bound state in the chiral quark model, Phys. Rev. D 98, 054022 (2018).

[79] H. Huang and J. Ping, Investigating tetraquarks composed of $u s \bar{d} \bar{b}$ and $u d \bar{s} \bar{b}$, Eur. Phys. J. C 79, 556 (2019).

[80] Y. Xing, F. S. Yu, and R. Zhu, Weak decays of stable open-bottom tetraquark by SU(3) symmetry analysis, Eur. Phys. J. C 79, 373 (2019).

[81] S. S. Agaev, K. Azizi, and H. Sundu, Decay modes of the scalar exotic meson $T_{b s ; \bar{u} \bar{d}}^{-}$, Phys. Rev. D 100, 094020 (2019).

[82] J. B. Cheng, S. Y. Li, Y. R. Liu, Y. N. Liu, Z. G. Si, and T. Yao, Spectrum and rearrangement decays of tetraquark states with four different flavors, Phys. Rev. D 101, 114017 (2020).

[83] R. Aaij et al. (LHCb Collaboration), A model-independent study of resonant structure in $B^{+} \rightarrow D^{+} D^{-} K^{+}$decays, arXiv:2009.00025. 
[84] R. Aaij et al. (LHCb Collaboration), Amplitude analysis of the $B^{+} \rightarrow D^{+} D^{-} K^{+}$decay, arXiv:2009.00026.

[85] M. Karliner and J. L. Rosner, First exotic hadron with open heavy flavor: $c s \bar{u} \bar{d}$ tetraquark, arXiv:2008.05993.

[86] R. Molina, T. Branz, and E. Oset, A new interpretation for the $D_{s 2}^{*}(2573)$ and the prediction of novel exotic charmed mesons, Phys. Rev. D 82, 014010 (2010).

[87] Q. F. Lü, D. Y. Chen, and Y. B. Dong, Masses of doubly heavy tetraquarks $T_{Q Q^{\prime}}$ in a relativized quark model, Phys. Rev. D 102, 034012 (2020).
[88] Q. F. Lü, D. Y. Chen, and Y. B. Dong, Masses of fully heavy tetraquarks $Q Q \bar{Q} \bar{Q}$ in an extended relativized quark model, Eur. Phys. J. C 80, 871 (2020).

[89] S. Godfrey and N. Isgur, Mesons in a relativized quark model with chromodynamics, Phys. Rev. D 32, 189 (1985).

[90] E. Hiyama, Y. Kino, and M. Kamimura, Gaussian expansion method for few-body systems, Prog. Part. Nucl. Phys. 51, 223 (2003).

[91] P. A. Zyla et al. (Particle Data Group), Review of particle physics, Prog. Theor. Exp. Phys. (2020), 083 C01. 